\title{
Discrimination of Earthquakes and Explosions in Southern Russia Using Regional High-Frequency Three-Component Data from the IRIS/JSP
}

\section{Caucasus Network}

\author{
by W.-Y. Kim, V. Aharonian, A. L. Lerner-Lam, and P. G. Richards
}

\begin{abstract}
High-frequency regional records from small earthquakes (magnitude $<4.5$ ) and comparable magnitude chemical explosions are analyzed to find a reliable seismic discriminant in southern Russia near Kislovodsk. The digital, three-component seismograms recorded during 1992 by the Caucasus Network operated by Lamont-Doherty Earth Observatory since 1991 in the distance ranges 15 to $233 \mathrm{~km}$ are used. Mean vertical-component $P g / L g$ spectral amplitude ratios in the band 8 to 18 $\mathrm{Hz}$ are about 1.3 and 3.2 for earthquakes and explosions, respectively, in this region. We find that the vertical-component $P g / L g$ spectral ratio in the frequency band 8 to $18 \mathrm{~Hz}$ serves quite well for classifying these events. A linear discriminant function analysis indicates that the $\mathrm{Pg} / \mathrm{Lg}$ spectral ratio method provides discrimination power with a total misclassification probability of about $7 \%$. The $P g / L g$ spectral ratios of rotated, three-component regional records improve the discrimination power of the spectral ratio method over the vertical-component $P g / L g$ ratios. Preliminary analysis indicates that distance-corrected vertical-component $P g / L g$ ratios improve the discrimination power by about $4 \%$ over uncorrected ratios. But we find that an even better discriminant is the $\mathrm{Pg} / \mathrm{Lg}$ spectral ratio of the three-component regional records corrected for the free-surface effect. In the frequency band 8 to $18 \mathrm{~Hz}$, the freesurface-corrected three-component $\mathrm{Pg} / \mathrm{Lg}$ spectral ratio provides discrimination power with a total misclassification probability of only $2.6 \%$. Free-surface-corrected and network-averaged $P g / L g$ spectral ratios provide transportability of the spectral ratio method to various regions worldwide.
\end{abstract}

\section{Introduction}

The discrimination of small earthquakes from large chemical explosions (from mines and quarries) based on seismic signals recorded at regional distances (10 to 1000 $\mathrm{km}$ ) is an important issue facing numerous regional seismic networks. The seismic discrimination problem becomes especially severe in an area with poorly known seismicity such as southern Russia near Kislovodsk. It would be extremely useful to have a reliable and robust criterion that could be used to discriminate earthquakes from explosions in the context of global seismic monitoring.

Figure 1 depicts the Caucasus Network operated during November 1991 through the summer of 1994 by LamontDoherty Earth Observatory and Experimental Methodological Expedition, Institute of Physics of the Earth, Obninsk, Russia, under the IRIS/Joint Seismic Program. The network consists of six stations around Kislovodsk in southern Russia. The network was deployed to record seismic events in the region to study wave propagation beneath the Caucasus mountains in particular and southern Russia and the Middle
East in general. The region covered by the network includes areas that differ greatly in their attenuation properties, as previously known from WWSSN stations south of the Caucasus (e.g., Kadinsky-Cade et al., 1981). The network also has provided abundant high-frequency signals from small events, both natural and man-made. Within a degree of the center of the network, over half of the events recorded can be associated with active quarries, or roughly 150 events per year. These data are useful for characterizing discriminants of chemical explosions from earthquakes, a critical issue facing the monitoring networks planned for the Comprehensive Test Ban Treaty (e.g., Stump et al., 1994).

At each station, the signals are recorded on a three-component set of intermediate-period sensors with a natural period, $T_{0}=5 \mathrm{sec}$ (Kinemetrics SV1/SH1). The sensors are situated 3 to $4 \mathrm{~m}$ below the ground surface, and the signals are digitized on site by a digitizer with 16-bit resolution, which after digital filtering provides a 60 samples per second data stream. Instrument response of each seismograph is 


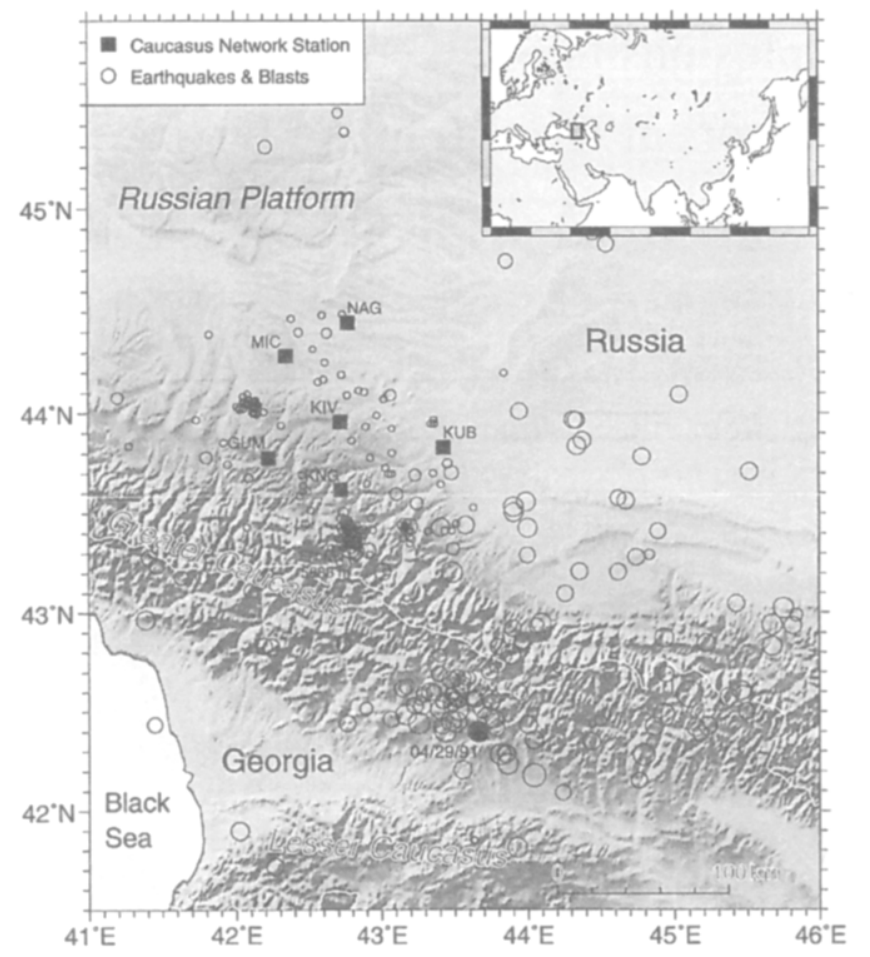

Figure 1. Locations of the Caucasus Network stations (solid squares) and near-regional $(\Delta<300 \mathrm{~km}$ ) seismic events (shaded circles) located by the Caucasus Network during 1992 are plotted on the topographic relief map. Notice the clusters of epicenters near $43.4^{\circ} \mathrm{N}: 42.8^{\circ} \mathrm{E}$ (Tyrnauz mine; Table 1 ), $44^{\circ}$ $\mathrm{N}: 42^{\circ}$ E (Ust-Djeguta quarry; Table 1), and $42.5^{\circ}$ $\mathrm{N}: 43.5^{\circ} \mathrm{E}$ (epicentral area of $04 / 29 / 91 M_{S}=7$ Georgian earthquake). Symbol sizes are proportional to the magnitudes of the events. Three major tectonic features in the region-Russian Platform, Greater Caucasus, and Lesser Caucasus-are indicated.

nearly flat to ground velocity in the frequency band 0.2 to $24 \mathrm{~Hz}$ (-3-db level). Digital signals from five remote stations are telemetered to a central recording site at Kislovodsk (KIV; Fig. 1), where they are recorded on magnetic tapes. Digital data recorded during 1992 are now available through the IRIS Data Management Center.

Most of the previous work on seismic discrimination has focused on separating large underground nuclear explosions from earthquakes in the context of a future comprehensive test ban (e.g., Pomeroy et al., 1982; Evernden et al., 1986; Taylor et al., 1989). In most earlier studies, data available for discrimination analyses were limited to frequencies below $10 \mathrm{~Hz}$, and previous work on regional signals from earthquakes and explosions in the western United States suggested that $P$ and $S$ waves from earthquakes often had stronger high frequencies (that is, up to $10 \mathrm{~Hz}$ ) than signals from explosions (e.g., Murphy and Bennett, 1982; Bennett and Murphy, 1986; Chael, 1988). There do not appear to be any robust discriminants based upon regional data for small events in the passband below $10 \mathrm{~Hz}$. However, at signifi- cantly higher frequencies, the $P g / L g$ spectral ratio shows great promise. Thus, Kim et al. (1993) reported that the mean $P g / L g$ spectral ratios in the band 1 to $25 \mathrm{~Hz}$ are about 0.5 and 1.25 for earthquakes and explosions, respectively, in the eastern United States $(\Delta \approx 10$ to $610 \mathrm{~km})$. Further, they found that the high-frequency $P g / L g$ spectral amplitude ratio in the frequency band 5 to $25 \mathrm{~Hz}$ was an adequate discriminant for classifying these events. In the eastern United States, the $P g / L g$ spectral amplitude ratio method provides discrimination power with a total misclassification probability of about $1 \%$ (Kim et al., 1993).

The regional signals from small seismic events in the northern Caucasus region are characterized by $\mathrm{Pg}$ and $\mathrm{Lg}$ onset arrivals with group velocities of about 5.8 and $3.2 \mathrm{~km} /$ $\mathrm{sec}$, respectively, at distances of a few tens of kilometers to about $200 \mathrm{~km}$. This $L g$ group velocity of $3.2 \mathrm{~km} / \mathrm{sec}$, which is somewhat slower than the global average of 3.4 to $3.5 \mathrm{~km} /$ sec, indicates lower than normal $S$-wave speeds in the crust around the Caucasus Network. Frequency content of the regional seismic signals from the earthquakes and quarry blasts may be best examined using spectrograms. Figure 2 shows spectrograms and corresponding time series from an earthquake and a quarry blast (multiple-hole instantaneous blast) at a comparable distance range from $\operatorname{KIV}(\Delta \approx 65$ $\mathrm{km})$. The earthquake record is characterized by a strong $S$ wave amplitude at low frequencies (below about $7 \mathrm{~Hz}$ ), and $P$ and $S$ waves with comparable amplitude at higher frequencies $(10$ to $15 \mathrm{~Hz})$. The spectrogram of the quarry blast record shows predominant $P$-wave energy over a wide frequency band 3 to $20 \mathrm{~Hz}$ and weak $S$ waves in this band as well as clear spectral banding due to source multiplicity (Fig. 2). The time series of these events also show clear differences between the earthquake and explosion records.

Although spectrograms can provide effective discrimination, their interpretation is usually a matter of expert judgment that cannot be turned into a simple, objective algorithm applicable on a routine basis to large numbers of events. However, recent developments suggest that spectrograms can be efficiently used in seismic discrimination by using an artificial neural network that can be trained to classify earthquakes from explosions (Dowla et al., 1990; Dysart and Pulli, 1990; Wang and Teng, 1995). Our observations of high-frequency ( 1 to $25 \mathrm{~Hz}$ ) regional signals from earthquakes and explosions in southern Russia indicate that these seismograms show distinctively different patterns in the spectral content of $P$ and $S$ signals, between earthquakes and explosions. This suggests that the $P g / L g$ spectral amplitude ratio can be used as a basis for an objective discriminant.

In the following sections, we report on our measurements of the $P g / L g$ spectral amplitude ratio, using high-frequency digital seismograms from earthquakes and chemical explosions recorded by the Caucasus Network (Fig. 1), and we evaluate their discrimination capability, paying particular attention to the improvements attainable with three-component records as compared to conventional $P g / L g$ spectral ratios based only on vertical-component data. 

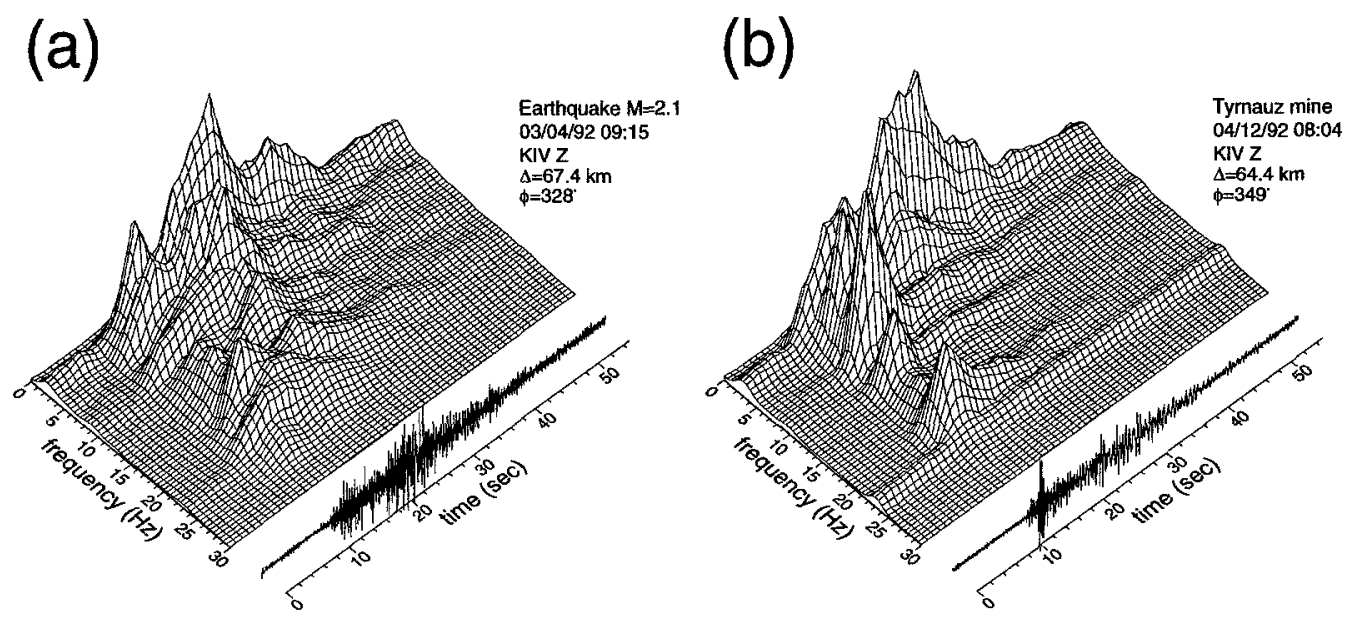

Figure 2. A comparison of frequency contents of the regional seismic signals from an earthquake and a ripple-fired quarry blast. (a) Spectrogram of the earthquake record shows strong $S$-wave amplitude at low frequencies (below about $5 \mathrm{~Hz}$ ), and at higher frequencies (10 to $15 \mathrm{~Hz}$ ), $P$ and $S$ waves have comparable amplitude. (b) Spectrogram of the quarry blast record shows predominant $P$-wave energy over a wide frequency band of 3 to $20 \mathrm{~Hz}$ but weak $S$ waves in this band. The spectrogram of earthquake record shows random distribution of seismic-wave energy, while the explosion signal shows clear spectral banding due to source multiplicity (ripple firing; a spectral band at about $22 \mathrm{~Hz}$ is due to noise).

\section{Data}

We present the analysis of digital seismograms from 25 explosions (presumably quarry blasts) and 25 earthquakes to obtain a specific discriminant. Most of the earthquakes are in the magnitude range 1 to 4 and are reported by the Joint Seismic Program Center (JSPC) or the U.S. Geological Survey (PDE). Seismicity during 1992, within a radius of about $200 \mathrm{~km}$ from station KIV, is plotted in Figure 1. We examined all earthquakes and quarry blasts that appear as clusters in Figure 1. Events are selected to sample a wide range of propagation paths around the network. All quarry blasts are from three known quarry sites. Locations of mines and quarries in the region are depicted in Figure 3 and listed in Table 1. Accurate locations of the Tyrnauz mine in the south of the network (Stump et al., 1994) as well as a Gypsum mine near the town of Zhako are provided by F. RiviéreBarbier (Center for Seismic Studies, personal comm., March 1994), while locations of other mines and quarries are obtained from a map provided by V. Khalturin (personal comm., March 1994). Locations of the Tyrnauz and Zhako mines obtained from different sources as noted above coincided within about $\pm 1 \mathrm{~km}$ (Table 1).

Only explosions for which we had information about the blast characteristics (usually blast time and location; Table 1) or events whose signals were clearly identified as quarry blasts are included in the sample data for the explosion group. Blast time is one of the most practical discriminants for large chemical explosions. About $87.5 \%$ of events, 77 out of 89 events recorded during 1992, located within the $15-\mathrm{km}$ radius from the Tyrnauz mine are clustered at two peak times (10 a.m. and 4 p.m. local time), while all 31 events located within the $10-\mathrm{km}$ radius from the Ust-Djeguta and Tsementny-Zavod quarries are clustered at 2 p.m. local time (see Fig. 1). Distance ranges of the data, 72 three-component records for 25 earthquakes and 62 three-component records for 25 explosions, are 21 to $233 \mathrm{~km}$ with means of 98 and $74 \mathrm{~km}$ for earthquakes and explosions, respectively. For vertical component, 82 and 78 records were available for earthquakes and explosions, respectively, for the events.

$P g$ and $L g$ signals are windowed with a Gaussian weighting function centered at group velocities around 5.6 and $3.2 \mathrm{~km} / \mathrm{sec}$, respectively. A standard deviation, $\sigma$, of $\sigma_{\text {ref }}$ $=2.5 \mathrm{sec}$ is used for the $L g$ Gaussian window at a reference distance of $\Delta_{\mathrm{ref}}=100 \mathrm{~km}$. The window includes the $\mathrm{Lg}$ arrivals with group velocities between 3.48 and $2.96 \mathrm{~km} / \mathrm{sec}$ in its $\pm \sigma$ width, and the Gaussian window is truncated at $\pm 1.96 \sigma(\approx 95 \%)$, which include signals within the group velocities 3.80 to $2.77 \mathrm{~km} / \mathrm{sec}$. The window lengths at different distances are scaled by $\sigma=\sigma_{\text {ref }} \times \Delta / \Delta_{\text {ref }}$. Thus, the time window used for $L g$ has the same group velocity window regardless of distance. The $P g$ window length is scaled to the $L g$ window using an average propagation velocity ratio $V_{s} / V_{p}(\approx 1 / \sqrt{3}) \times L g$ window length, so that $P g$ crustal paths similar to the paths making up $L g$ are included.

The $P g$ and $L g$ signals, weighted by the Gaussian functions, are fast Fourier transformed. The resulting amplitude spectra are smoothed with another Gaussian function having $\sigma=1 \mathrm{~Hz}$ and are resampled at every $2-\mathrm{Hz}$ interval from 2 to $24 \mathrm{~Hz}$. Noise analyses indicate that the signal-to-noise $(\mathrm{S} / \mathrm{N})$ ratios are quite high in most of the records, but in some cases, the signals fall to the background noise level above 


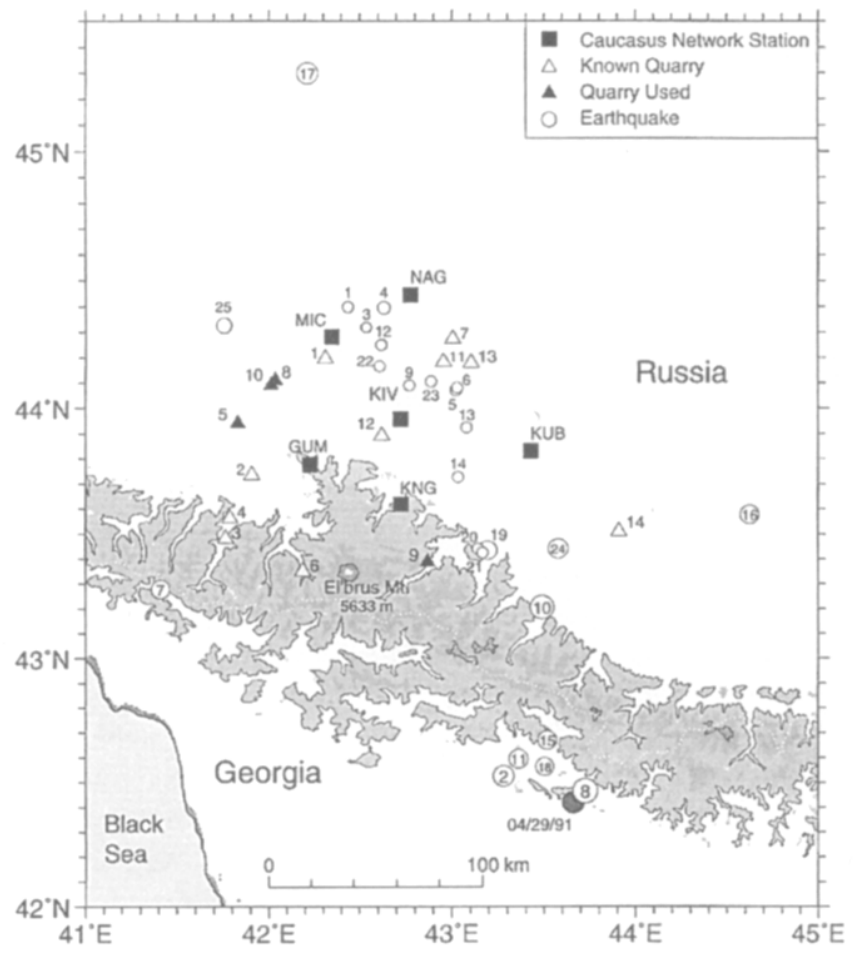

Figure 3. Locations of earthquakes (circles), mines, and quarries identified and used in this study (solid triangles), other known quarries (open triangles), and Caucasus Network stations (solid squares). Shaded region represents areas over $2000 \mathrm{~m}$ elevation topped by El'brus Mt. (5633 m), which is the highest peak in Europe west of the Urals. Circle size is proportional to the magnitude of the earthquakes. Numbers for the quarries and earthquakes refer to the event IDs given in Tables 1 and 2 .

Table 1

Quarries and Mines around Kislovodsk, Russia*

\begin{tabular}{llcccccc}
\hline $\begin{array}{c}\text { Id } \\
\#\end{array}$ & \multicolumn{1}{c}{$\begin{array}{c}\text { Quarry } \\
\text { Name }\end{array}$} & $\begin{array}{c}\text { Lat. } \\
\left({ }^{\circ} \mathrm{N}\right)\end{array}$ & $\begin{array}{c}\text { Long. } \\
\left({ }^{\circ} \mathrm{E}\right)\end{array}$ & $\begin{array}{r}\text { Id } \\
{ }^{*}\end{array}$ & $\begin{array}{c}\text { Quarry } \\
\text { Name }\end{array}$ & $\begin{array}{c}\text { Lat. } \\
\left({ }^{\circ} \mathrm{N}\right)\end{array}$ & $\begin{array}{c}\text { Long. } \\
\left({ }^{\circ} \mathrm{E}\right)\end{array}$ \\
\hline 1 & Alenovsky & 44.20 & 42.31 & 8 & $\begin{array}{c}\text { Tsementny- } \\
\text { Zavod }\end{array}$ & 44.11 & 42.04 \\
& & & & & & \\
2 & Andezit & 43.74 & 41.91 & 9 & Tyrnauz $\dagger$ & 43.39 & 42.87 \\
3 & Byely-Mramoz & 43.48 & 41.76 & 10 & Ust-Djeguta & 44.09 & 42.02 \\
4 & Cher-Mramoz & 43.56 & 41.79 & 11 & Verblud & 44.18 & 42.96 \\
5 & Zhako $\dagger$ & 43.94 & 41.83 & 12 & Zamok & 43.89 & 42.62 \\
6 & Grznitny & 43.35 & 42.19 & 13 & Zmeika & 44.18 & 43.11 \\
7 & Kinjal & 44.27 & 43.01 & 14 & Unidentified & 43.50 & 43.91 \\
\hline
\end{tabular}

*Based on local draft map (Dr. Vitaly Khalturin, personal comm., 1994). $\uparrow$ F. Riviére-Barbier, Center for Seismic Studies, Washington, D.C. (personal comm., March 1994).

about $20 \mathrm{~Hz}$. The $\mathrm{S} / \mathrm{N}$ ratio becomes less than 2 at about 20 $\mathrm{Hz}$ for records from distant events $(\Delta>150 \mathrm{~km})$. The $\log _{10}(P g / L g)$ spectral amplitude ratios at discrete frequency points are obtained for each record. Network-averaged $\log _{10}(P g / L g)$ ratios are then obtained for each event by averaging the discrete frequency values from all stations. Figure 4 shows the network-averaged $\log _{10}(P g / L g)$ ratios at discrete frequency points for 25 earthquakes and 25 explosions. Earthquake and explosion populations are well separated in the frequency band 6 to $18 \mathrm{~Hz}$ (Fig. 4). At frequencies lower than $6 \mathrm{~Hz}$, there is some overlap. Thus, the frequency band 6 to $18 \mathrm{~Hz}$ is the most reliable, and a sampled version will be used in the following discrimination analysis.

\section{The Linear Discriminant Function}

To test the discriminant power of the high-frequency $P g / L g$ spectral amplitude ratio, we performed multivariate discriminant analysis on vertical-component $\log _{10}(P g / L g)$ spectral ratios for the data set of earthquakes and explosions. The discriminant power is evaluated by developing an optimal classification rule that separates earthquakes from explosions based on the sample data we have on each group. Each sample data set of 25 events (i.e., 25 explosions and 25 earthquakes) is described by a matrix of six rows $\left[\log _{10}(P g / L g)\right.$ at $2-\mathrm{Hz}$ frequency intervals from 8 to $18 \mathrm{~Hz}$ ] and 25 columns. Preliminary analysis suggested somewhat higher $P g / L g$ ratios from Tyrnauz mine blasts than for other quarry blasts, but we merged both data sets into one explosion group because the differences between them were much smaller than their differences from earthquakes.

We introduce $f_{\mathrm{Ex}}(\mathbf{r})$ and $f_{\mathrm{Eq}}(\mathbf{r})$ as the probability densities of the two types of events (explosions and earthquakes), with $\mathbf{r}$ as a column vector representing the $\log$ spectral ratios sampled at six values. We take $\pi_{\mathrm{Ex}}$ and $\pi_{\mathrm{Eq}}$ as the a priori probability of the two types of events, so $\pi_{\mathrm{Ex}}+\pi_{\mathrm{Eq}}=1$. We follow standard practice by assigning an event to the earthquake class if

$$
f_{\mathrm{Eq}}(\mathbf{r}) / f_{\mathrm{Ex}}(\mathbf{r})>\pi_{\mathrm{Ex}} / \pi_{\mathrm{Eq}}
$$

and to the explosion class otherwise (see, e.g., Seber, 1984, for multivariate discrimination methods). This rule is optimum, in that it minimizes the total probability of misclassification. Our knowledge of $f_{\mathrm{Ex}}(\mathbf{r})$ and $f_{\mathrm{Eq}}(\mathbf{r})$ comes from the sample data set, and we introduce and evaluate a linear discriminant function $D(\mathbf{r})$ under the assumptions that (a) the sample distributions are normal, (b) the dispersion (variance-covariance) matrices of the two groups are the same, and (c) the sample data observations are correctly classified. Then the linear function that best separates the two types of seismic source is

$$
D(\mathbf{r}) \equiv \lambda^{\mathrm{T}}\left[\mathbf{r}-\left(\boldsymbol{\mu}_{\mathrm{Eq}}+\boldsymbol{\mu}_{\mathrm{Ex}}\right) / 2\right]
$$

where $\boldsymbol{\mu}_{\mathrm{Eq}}$ is the mean of the earthquake sample data values $\mathbf{r}_{\mathrm{Eq}}^{i}(i=1, \ldots, 25)$, and similarly for $\boldsymbol{\mu}_{\mathrm{Ex}}, \lambda=\mathbf{S}^{-1}\left(\boldsymbol{\mu}_{\mathrm{Eq}}-\right.$ 


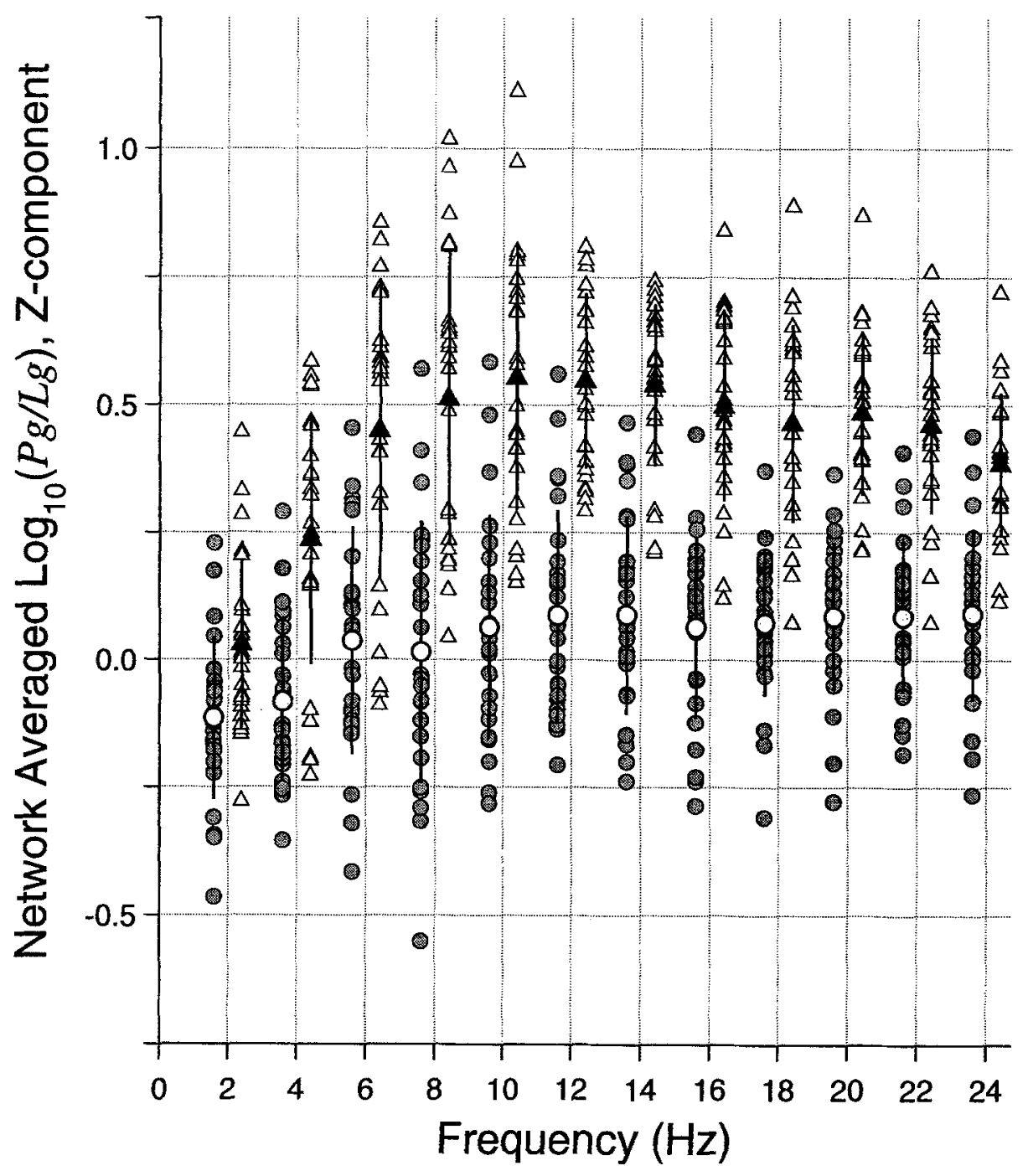

Figure 4. Network-averaged vertical-component $\log _{10}(P g / L g)$ spectral amplitude ratios at 12 discrete frequency points used in discrimination analysis are plotted for earthquakes (shaded circles) and explosions (open triangles). A mean value of each discrete frequency point is plotted for earthquakes (open circles) and explosions (solid triangles) with their arms representing the scatter (S.D.).

$\left.\boldsymbol{\mu}_{\mathrm{Ex}}\right) ; \mathrm{S}$ is the average of the dispersion matrices $\mathrm{S}_{\mathrm{Eq}}$ and $\mathrm{S}_{\mathrm{Ex}}$, with (for example) $\mathbf{S}_{\mathrm{Eq}}=1 / 24 \sum_{i=1}^{25}\left(\mathbf{r}_{\mathrm{Eq}}^{i}-\boldsymbol{\mu}_{\mathrm{Eq}}\right)^{\mathrm{T}}\left(\mathbf{r}_{\mathrm{Eq}}^{i}-\boldsymbol{\mu}_{\mathrm{Eq}}\right)$; and superscript $\mathbf{T}$ denotes a transpose. Equation (2) defines a hyperplane for separating the two groups.

In terms of these easily calculated quantities, the discrimination analysis is very simple. Rule (1) becomes the following: Assign an event $\mathbf{r}$ to the earthquake population if $D(\mathbf{r})>\ln \left(\pi_{\mathrm{Eq}} / \pi_{\mathrm{Ex}}\right)$. If $\pi_{\mathrm{Eq}}=\pi_{\mathrm{Ex}}=1 / 2$ (i.e., earthquakes and explosions have equal a priori probabilities), the rule is even simpler: The event is labeled an earthquake or an explosion according to $D(\mathbf{r})>0$ or $D(\mathbf{r})<0$. In this case, we have

$$
\begin{aligned}
P(\text { misclassification })= & \frac{1}{\sqrt{2 \pi}} \int_{-\infty}^{-\Delta / 2} \exp \left(-x^{2} / 2\right) d x \\
& \text { where } \Delta^{2}=\lambda^{\mathrm{T}}\left(\mu_{\mathrm{Eq}}-\mu_{\mathrm{Ex}}\right)
\end{aligned}
$$

for the probability of misclassification. $\Delta^{2}$ is the Mahalanobis $D$-squared measure of distance between the two means.

This type of multivariate analysis was developed and used by Fisher (1936) and has been used in many seismological applications-for example, by Jebe and Willis (1964), Sandvin and Tjøstheim (1978), and Kim et al. (1993). The equality of the dispersion matrices for the two groups is tested using a $\chi^{2}$ test (Seber, 1984). If the dispersion matrices of the populations are not the same, then a 
quadratic discriminant function is appropriate (Davis, 1986; Seber, 1984).

\section{Applications of Discriminant Analysis}

High-Frequency Network-Averaged

Vertical-Component $P g / L g$ Ratio

The sample data sets consisting of 25 earthquakes and 25 explosions were analyzed using the linear discriminant function given in (2). For each event, network-averaged vertical-component $\log _{10}(P g / L g)$ ratios at frequencies of 8,10 , $12,14,16$, and $18 \mathrm{~Hz}$ correspond to the variables $r_{1}, r_{2}, r_{3}$, $r_{4}, r_{5}$, and $r_{6}$. The linear discriminant function obtained is

$$
\begin{aligned}
D(\mathbf{r})=6.68+10.04 r_{1}-8.22 r_{2}-2.03 r_{3} \\
-15.53 r_{4}+6.14 r_{5}-11.15 r_{6},
\end{aligned}
$$

and the Mahalanobis $D$-squared measure is $\Delta^{2}=8.67$. Assuming equal prior probabilities for the two groups, we assign event $\mathbf{r}$ to the earthquake class if $D(\mathbf{r})>0$. Applying this rule to the earthquake and explosion data, we find that three earthquakes and an explosion are classified incorrectly, and the misclassification probability from equation (3) is 0.071 . Values of $D(\mathbf{r})$ may be called the discriminant score and are plotted in Figure 5 with respect to the mean $\log _{10}(P g /$ $L g$ ) spectral amplitude ratio of each event. Vertical lines in the figure denoted as $\mathrm{Eq}$ and $\mathrm{Ex}$ are the projection of the multivariate mean of the earthquake and explosion populations, respectively. The vertical line, $D_{0}$, is the line $D(\mathbf{r})=$ 0 , which serves to classify the events when the a priori probability of the two populations is the same. The distance between $\mathrm{Eq}$ and $\mathrm{Ex}$ is the Mahalanobis $D$-squared measure of distance between two populations, since, from (2), $D\left(\mu_{\mathrm{Eq}}\right)$ $-D\left(\mu_{\mathrm{Ex}}\right)=\Delta^{2}$. It is shown in Figure 5 that all the earthquake records from various paths in southern Russia near Kislovodsk have a mean vertical-component $P g / L g$ spectral ratio of about 1.3, while the explosion records show a mean of about 3.2 in the frequency bands 8 to $18 \mathrm{~Hz}$. The locations of three misclassified earthquakes-events 8,11 , and 16 (see Table 2)-are indicated in Figure 5.

\section{Network-Averaged Three-Component $P g / L g$ Ratio}

The Caucasus Network and many other modern seismograph stations are now equipped with three-component broadband sensors. Although high-frequency regional signals on the vertical component show reasonable discrimination power for classifying earthquakes from explosions, there is interest in the evaluation of three-component records to see if they can provide improved discrimination. We have therefore examined $P g / L g$ spectral ratio methods to discriminate the earthquakes from explosions using regional threecomponent records. Our first approach was simply to rotate the observed N-S- and E-W-component seismogram pairs to obtain radial $(R)$ and tangential $(T)$ components. Then the $P g / L g$ ratios of three-component records are formed for each station by defining

$$
\frac{P g}{L g}=\frac{\sqrt{P g_{Z}^{2}+P g_{R}^{2}}}{\sqrt{L g_{Z}^{2}+L g_{R}^{2}+L g_{T}^{2}}},
$$

where subscripts indicate the component. For example, $P g_{Z}$ indicates spectral amplitude of $P$ waves (mostly $P g$ ) on vertical component $(Z)$, and $L g_{R}$ indicates spectral amplitude of $L g$ waves on radial component. A single three-component $\mathrm{Pg} / \mathrm{Lg}$ ratio is obtained for each station from equation (5) and the network mean $\log _{10}(P g / L g)$ is obtained by averaging over stations for each event. Figure 6 shows the networkaveraged $\log _{10}(\mathrm{Pg} / \mathrm{Lg})$ ratios at discrete frequency points for 25 earthquakes and 25 explosions. Earthquake and explosion populations are better separated in the frequency bands 2 to 4 and 12 to $20 \mathrm{~Hz}$ than the vertical-component ratios shown in Figure 4. There is also a clear difference between the spectral ratios at low frequencies ( 2 to $10 \mathrm{~Hz}$ ) and high frequencies $(12$ to $20 \mathrm{~Hz}$ ) within the same group, in particular, for earthquake data (Fig. 6). The network-averaged $\log _{10}(P g / L g)$ ratios of the three-component records from the earthquakes along various paths in southern Russia have a mean three-component $P g / L g$ spectral ratio of about 0.73 , while the explosion records show a mean of about 2.2. These mean $P g / L g$ ratios are slightly lower, by about a factor of 1.6, than the vertical-component data shown earlier (see Fig. 4), mainly due to the inclusion of $L g$ waves on radial and tangential components ( $L g$ is typically stronger on these components than on the vertical component).

We repeated the multivariate analysis using three-component $P g / L g$ ratios. The discriminant scores of each event are plotted with respect to the network mean $\log _{10}(P g / L g)$ spectral amplitude ratio in Figure 7 and are listed in Tables 2 and 3. Discriminant function analyses for the three-component data suggest that the use of three-component records reduces the scatter of the mean $\log _{10}(P g / L g)$. The Mahalanobis $D$-squared measure is $\Delta^{2}=14.07$, indicating a greater separation between earthquake and explosion populations than was the case with vertical-component data. Three distant earthquakes, events 8, 11, and 16 (see Fig. 3), that were misclassified in the vertical-component analysis are now correctly classified. In general, distant earthquakes show higher mean $\log _{10}(P g / L g)$ than near events (Figs. 3 and 7). Most of the Tyrnauz mine explosions have higher mean spectral ratios than signals from the other two mining areas (Fig. 7).

The discriminant function analysis indicates that all events in the sample data are classified correctly and the total misclassification probability is only $3.04 \%$, suggesting that the three-component $P g / L g$ ratios improve the discrimination power of high-frequency regional data by about $4 \%$ over the use of single vertical-component $P g / L g$ ratios $(92.9 \%$ versus $97.0 \%$ ).

\section{Removal of Free-Surface Effect on Regional Records}

Although high-frequency $P g / L g$ spectral ratios of regional signals on vertical-component records show reason- 


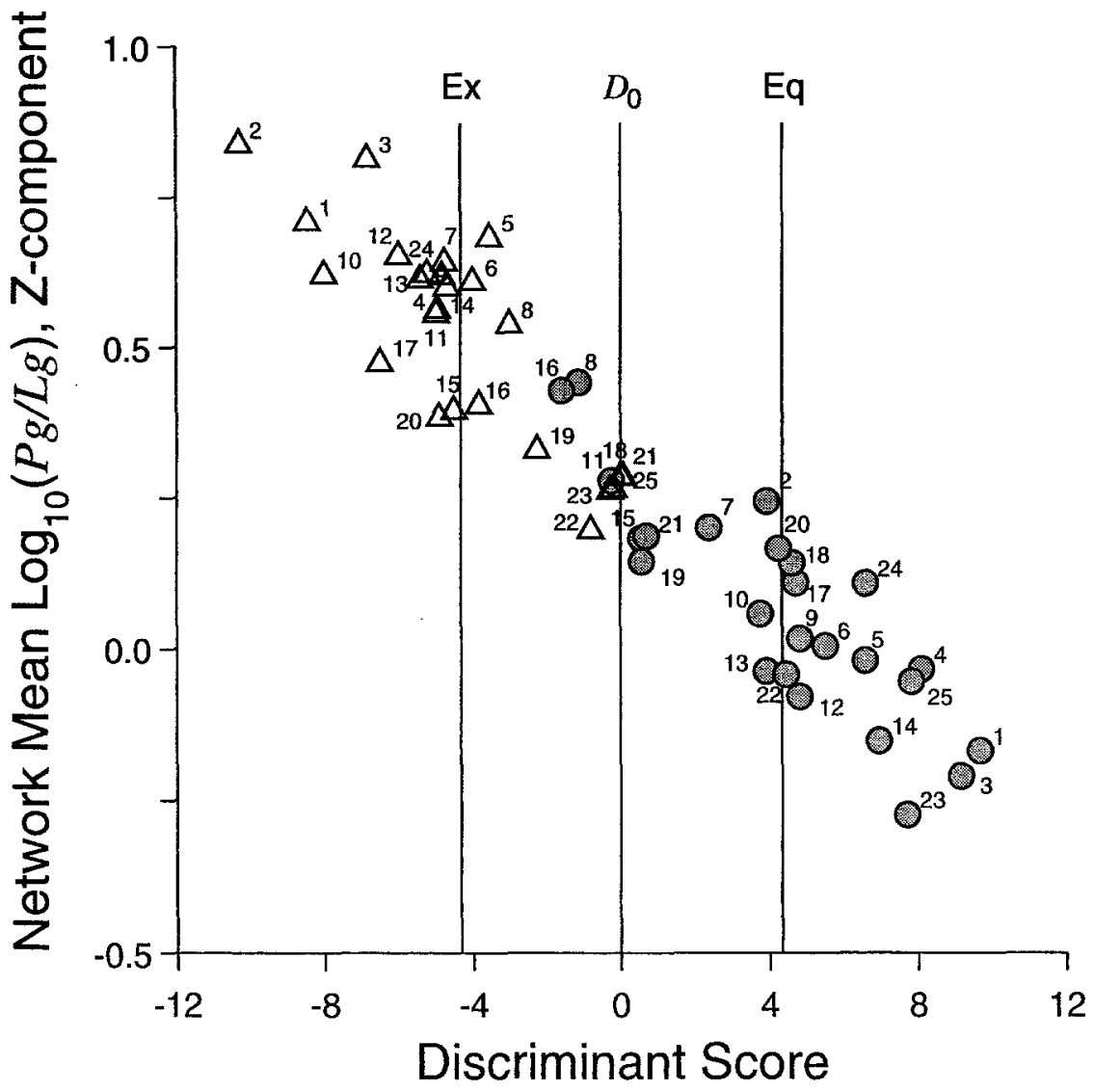

Figure 5. Discriminant scores of earthquakes (shaded circles) and explosions (open triangles) of the sample data are plotted with their mean network-averaged $\log _{10}(P g /$ $L g$ ) ratios. Note that the two populations are also separated by mean vertical-component $\log _{10}(P g / L g)$ ratio $\approx 0.25$. Vertical lines denoted as Ex and Eq are the projection of the multivariate means of the earthquake and explosion population, respectively. The vertical line $D_{0}$ is the classification line. Two earthquakes and an explosion are incorrectly classified, and the total misclassification probability is $7.1 \%$.

able discrimination power for classifying earthquakes from explosions-and rotated three-component records are apparently a significant improvement-the success of such discriminants in trial regions does not always warrant their applicability to various other regions worldwide. The key to the successful discrimination of various types of seismic sources is the clear observation of the signals radiated from the seismic sources. One of the most important problems is correction of the effects of the source-receiver paths on observed regional signals. The source-receiver path effects include, for example, attenuation along the paths, different geometrical spreading of $P$ and $S$ waves, local receiver site responses, and interaction of the incoming wave field with the free surface.

The effect of the free surface in the neighborhood of the receiver on the recorded regional signals is significant, and its main effects are amplification of incoming waves. However, unlike other path effects, it can be removed by a relatively simple real transformation (House and Boatwright, 1980; Kennett, 1991). Recently, Kennett (1991) showed that a set of approximate free-surface correction operators can be formed to remove the free-surface effects over the slowness bands for the main regional phases.

For a $P$ wave, with pulse shape $P(t)$ and particle motion having $(x, y, z)$ components $P(t)(\sin i, 0,-\cos i)$, incident upon a free surface perpendicular to the $z$ (depth) direction, the motion of the free surface itself is (Aki and Richards, 1980; p. 190)

$$
\begin{gathered}
P(t)\left[\frac{4 \alpha p}{\beta^{2}} \frac{\cos i}{\alpha} \frac{\cos j}{\beta}, 0, \frac{-2 \alpha}{\beta^{2}} \frac{\cos i}{\alpha}\left(\frac{1}{\beta^{2}}-2 p^{2}\right)\right] \\
\left(\frac{1}{\beta^{2}}-2 p^{2}\right)^{2}+4 p^{2} \frac{\cos i}{\alpha} \frac{\cos j}{\beta} \\
=[R(t), 0,-Z(t)],
\end{gathered}
$$

where $\alpha=$ surface $P$-wave velocity, $\beta=$ surface $S$-wave velocity, $i=$ angle of incidence of the $P$ wave, $j=$ angle of incidence of the $S$ wave, $p=$ slowness, and $\sin i / \alpha=$ $\sin j / \beta=p$. Note that $Z(t)$ is conventionally measured positive upward, opposite to the depth direction; and $R(t)$ is positive away from the source. The ground motions in a 


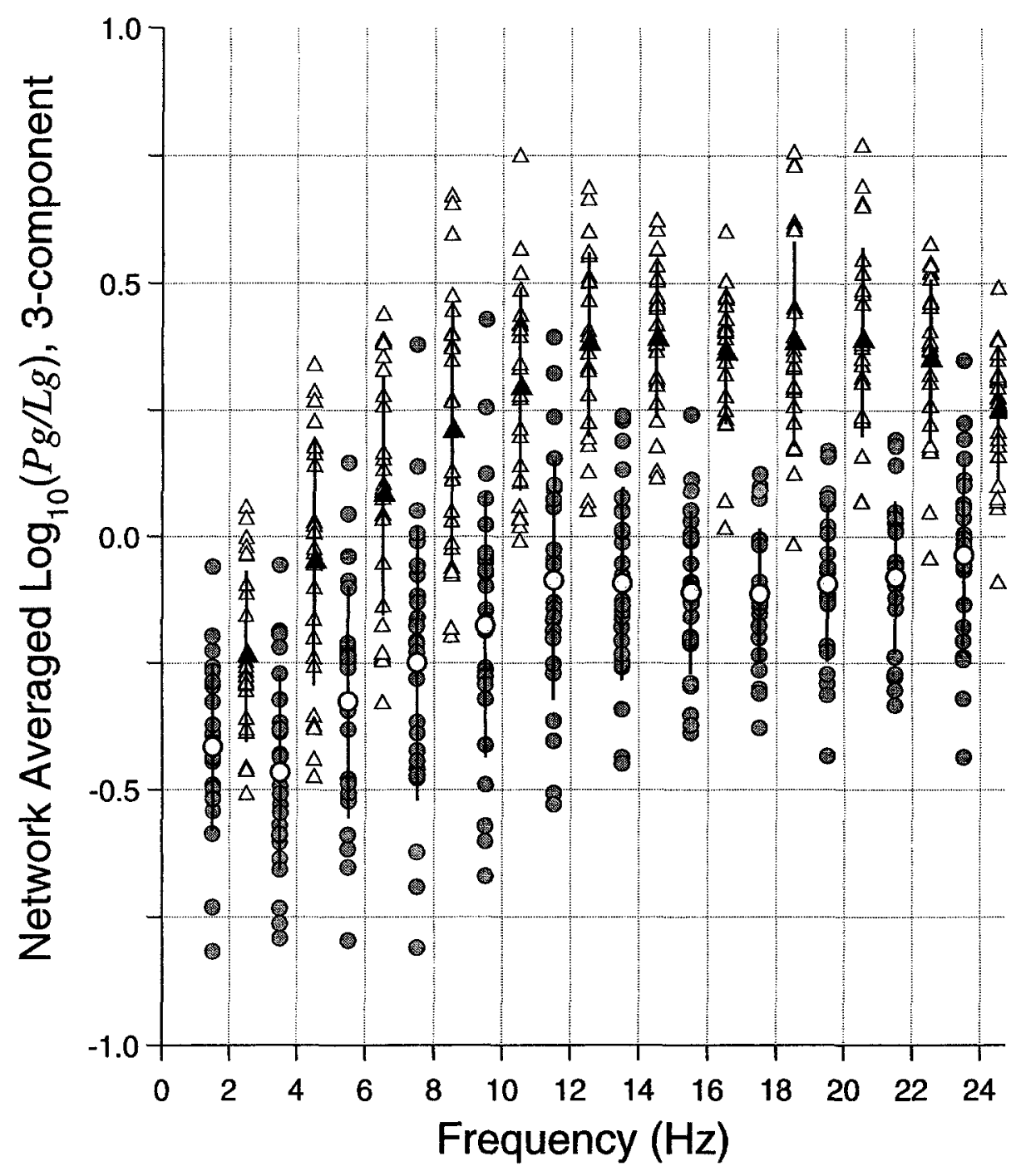

Figure 6. Network-averaged three-component $\log _{10}(P g / L g)$ spectral amplitude ratios at $\mathbf{1 2}$ discrete frequency points used in discrimination analysis are plotted for earthquakes (shaded circles) and explosions (open triangles). Other notation is the same as in Figure 4.

homogeneous half-space for a $P$ wave incident upon a free surface are depicted in Figure 8.

The motion of the incident $P$ and $S V$ wave can then be recovered as a linear combination of the radial, $R(t)$, and vertical, $Z(t)$, components available from three-component records. Thus, equation (6) can be used to show that

$$
P(t)=\frac{\cos 2 j}{2 \cos i} \cdot Z(t)+\frac{\beta}{\alpha} \sin j \cdot R(t)
$$

The corresponding result for an incident $S V$ wave is

$$
S V(t)=\frac{\cos 2 j}{2 \cos j} \cdot R(t)-\sin j \cdot Z(t)
$$

which has long been used by those studying earthquake source functions to infer the $S$-wave pulse shape from threecomponent data (House and Boatwright, 1980).

The incident $S H$ wave is simply one-half of the recorded tangential component:

$$
S H(t)=\frac{1}{2} T(t)
$$

If the incident $P$ wave is contaminated by $S V$ motion due to local horizontal layering, then equation (7a) gives precisely the right combination of $R$ and $Z$ to eliminate the contribution from $S V$. Similarly, (7b) removes contaminating $P$ (due to horizontal layering) from the $S V$ trace.

An example of three-component records of an explosion from the Tyrnauz mine recorded at station MIC $(\Delta=108$ $\mathrm{km}$ ) is shown in Figure 9. The rotated seismograms, $Z, R$, and $T$ components, show that $P$ waves on the $Z$ component 


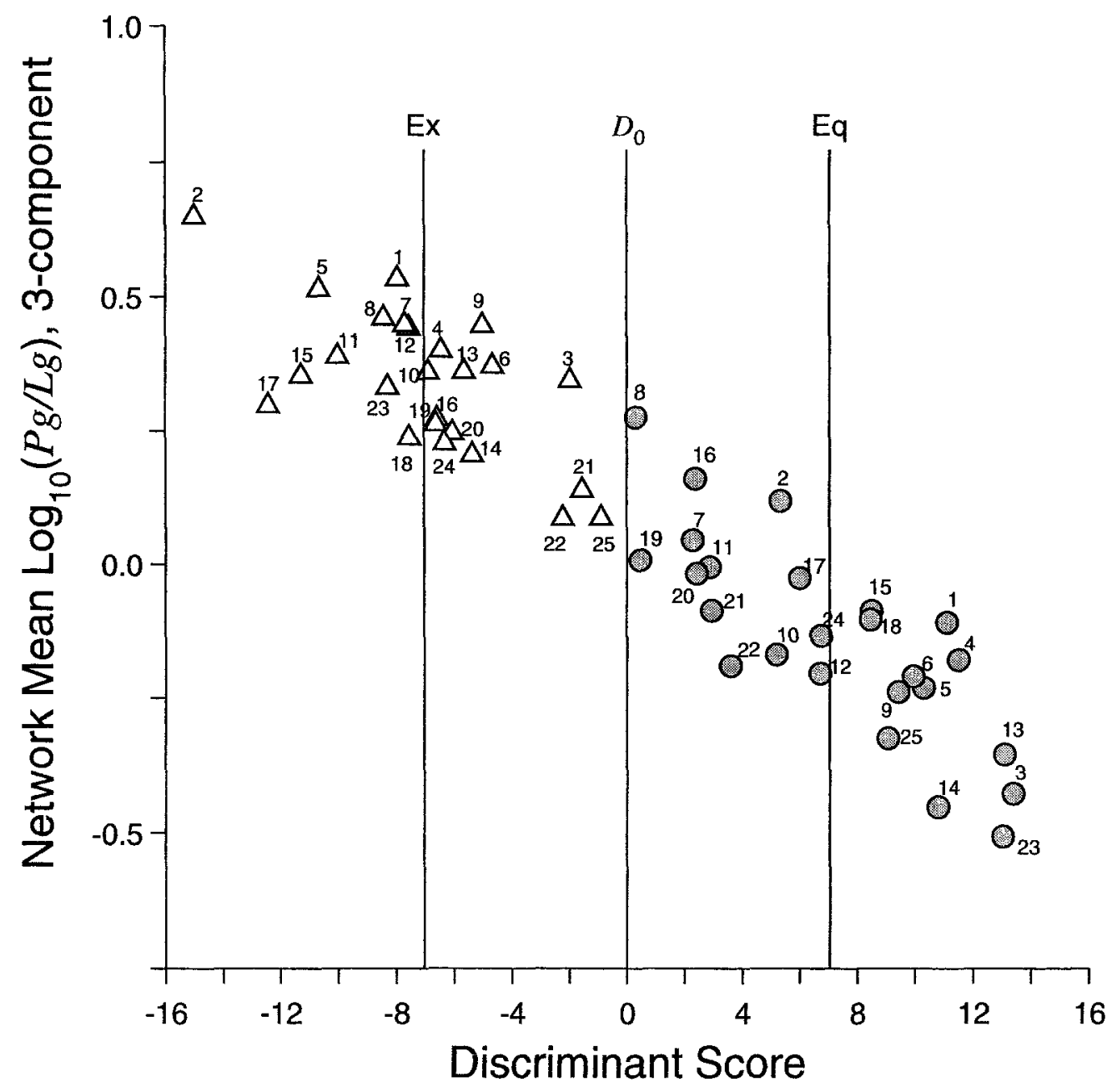

Figure 7. Discriminant scores of earthquakes (shaded circles) and explosions (open triangles) of the sample data are plotted with their mean network-averaged $\log _{10}(P g /$ $L g$ ) ratios. All earthquakes and explosions are correctly classified, and the total misclassification probability is $3.0 \%$. Each event is indicated by its event ID numbers given in Tables 2 and 3 and in Figure 3. Notice that distant earthquakes, numbers 2, 8, and 16 , have relatively high spectral ratios. Other notation is the same as in Figure 5.

as well as $L g$ waves on the $R$ and $T$ components have comparable amplitudes (Fig. 9). However, when free-surface effects are removed from the rotated seismograms by successively applying free-surface correction operators appropriate for major regional phases $(P n, P g$, and $L g)$, the recovered incident wave-vector estimates allow us to compare directly the $P$-, $S V$-, and $S H$-wave amplitudes on different traces.

The composite incident wave-vector traces in Figure 9 are obtained by applying transformations (equations $7 \mathrm{a}$ through $7 \mathrm{c}$ ) sequentially to successive group velocity windows along the trace. Successive windows used are indicated in Figure 9 with corresponding slownesses. Assuming $\alpha=$ $4.5 \mathrm{~km} / \mathrm{sec}$ and $\beta=2.6 \mathrm{~km} / \mathrm{sec}$ appropriate for the region, signals with group velocities greater than $5.2 \mathrm{~km} / \mathrm{sec}$ are transformed with an approximate $P n$ slowness, $p=0.08$ $\mathrm{sec} / \mathrm{km}\left(i \approx 25^{\circ}\right)$; signals with group velocities 5.2 to $4 \mathrm{~km} /$ sec are transformed with an approximate $P g$ slowness, $p=$
$0.14 \mathrm{sec} / \mathrm{km}\left(i \approx 40^{\circ}\right)$; and signals with group velocities 3.3 to $2.6 \mathrm{~km} / \mathrm{sec}$ are transformed with an approximate $L g$ phase slowness, $p=0.34 \mathrm{sec} / \mathrm{km}\left(j \approx 60^{\circ}\right)$. The $S V$ transformation is sensitive to the slowness used, since the free-surface correction factor for $R$ component in (7b) varies rapidly for slownesses $1 /(\sqrt{2} \beta)<p<1 / \beta$. When the precise slownesses of the incoming waves are not available for correct transformation, our experience suggests that a reasonable choice of the slowness is when the $P$ wave on the $S V$ trace after the transformation has comparable amplitude to the $P$ wave on the $S H$ trace, since $P$ waves on both traces are derived from scattering, for example, from heterogeneities other than horizontal layering. Similarly for $\mathrm{Lg}$ signals from the explosions, a reasonable choice of the slowness is when the $L g$ waves on the $P$ trace are comparable to the $L g$ waves on the $S H$ trace. For $p>1 / \alpha$, the coefficient $(\cos 2 j) /(2 \cos i)$ is complex, since $P$ waves become evanescent and $\cos i=(1$ 
Table 2

List of Earthquakes Used in This Study

\begin{tabular}{|c|c|c|c|c|c|c|c|c|c|c|}
\hline \multirow{2}{*}{$\begin{array}{l}\text { Id } \\
\#\end{array}$} & \multicolumn{2}{|c|}{ Origin Time } & \multirow{2}{*}{$\begin{array}{l}\text { Lat. } \\
\left({ }^{\circ} \mathrm{N}\right)\end{array}$} & \multirow{2}{*}{$\begin{array}{l}\text { Long. } \\
\left({ }^{\circ} \mathrm{E}\right)\end{array}$} & \multirow{2}{*}{$\begin{array}{l}\text { Depth } \\
(\mathrm{km})\end{array}$} & \multirow{2}{*}{$\begin{array}{c}m_{b} \\
(\mathrm{JSPC})^{*}\end{array}$} & \multirow{2}{*}{$\begin{array}{c}\text { Distance } \\
(\mathrm{km})\end{array}$} & \multicolumn{3}{|c|}{ Three Component } \\
\hline & (yx mo dy) & (hh:mm:sec) & & & & & & Score & $P g / L g$ & $N$ \\
\hline 1 & 920216 & $13: 43: 14.8$ & 44.398 & 42.438 & 16 & 0.5 & 40.7 & 11.10 & -0.11 & 2 \\
\hline 2 & 920220 & $20: 18: 55.1$ & 42.526 & 43.280 & & 3.4 & 197.2 & 5.34 & 0.12 & 3 \\
\hline 3 & 920226 & $06: 13: 28.9$ & 44.317 & 42.536 & 18 & -0.2 & 53.9 & 13.38 & -0.43 & 2 \\
\hline 4 & 920228 & $01: 16: 53.7$ & 44.394 & 42.632 & 18 & 0.9 & 50.3 & 11.50 & -0.18 & 3 \\
\hline 5 & 920307 & $03: 06: 42.9$ & 44.069 & 43.024 & 30 & & 43.6 & 10.31 & -0.23 & 4 \\
\hline 6 & 920307 & 03:08:14.0 & 44.075 & 43.028 & 11 & & 43.6 & 9.95 & -0.21 & 4 \\
\hline 7 & 920322 & $05: 10: 00.9$ & 43.279 & 41.411 & & 2.9 & 144.6 & 2.27 & 0.05 & 3 \\
\hline 8 & 920327 & $19: 21: 04.8$ & 42.466 & 43.725 & 33 & $4.8 \dagger$ & 217.7 & 0.32 & 0.27 & 3 \\
\hline 9 & 920328 & $09: 39: 04.8$ & 44.089 & 42.770 & 12 & & 48.8 & 9.42 & -0.24 & 4 \\
\hline 10 & 920407 & $20: 31: 09.7$ & 43.206 & 43.491 & 15 & 4.5 & 134.5 & 5.18 & -0.17 & 2 \\
\hline 11 & 920409 & $17: 34: 00.9$ & 42.594 & 43.361 & & 2.8 & 175.0 & 2.88 & -0.01 & 3 \\
\hline 12 & 920412 & $02: 16: 30.9$ & 44.250 & 42.617 & 15 & 0.0 & 45.2 & 6.70 & -0.20 & 3 \\
\hline 13 & 920412 & $20: 00: 56.2$ & 43.923 & 43.081 & 28 & -0.1 & 43.1 & 13.11 & -0.36 & 3 \\
\hline 14 & 920416 & $14: 18: 57.5$ & 43.727 & 43.035 & 6 & & 60.4 & 10.80 & -0.45 & 3 \\
\hline 15 & 920509 & $20: 45: 06.8$ & 42.670 & 43.519 & & 2.9 & 173.6 & 8.50 & -0.09 & 3 \\
\hline 16 & 920621 & $21: 14: 24.6$ & 43.577 & 44.625 & 31 & 2.8 & 129.3 & 2.38 & 0.16 & 2 \\
\hline 17 & 920705 & $17: 34: 07.0$ & 45.298 & 42.212 & 13 & 3.8 & 149.8 & 6.01 & -0.03 & 3 \\
\hline 18 & 920711 & $14: 39: 17.8$ & 42.563 & 43.505 & & 2.6 & 184.8 & 8.47 & -0.10 & 3 \\
\hline 19 & 920716 & $17: 15: 33.3$ & 43.433 & 43.194 & & 2.9 & 91.2 & 0.47 & 0.01 & 3 \\
\hline 20 & 920716 & $17: 20: 09.6$ & 43.440 & 43.141 & & & 98.9 & 2.43 & -0.02 & 2 \\
\hline 21 & $9207 \quad 17$ & $19: 42: 03.7$ & 43.423 & 43.166 & 8 & & 77.5 & 2.95 & -0.09 & 2 \\
\hline 22 & 920723 & $01: 07: 02.8$ & 44.164 & 42.609 & 10 & & 37.1 & 3.59 & -0.19 & 3 \\
\hline 23 & 920723 & $01: 13: 15.0$ & 44.104 & 42.890 & 17 & 0.1 & 42.5 & 13.04 & -0.51 & 2 \\
\hline 24 & 920812 & $13: 23: 18.6$ & 43.440 & 43.578 & 22 & 3.3 & 94.6 & 6.75 & -0.13 & 4 \\
\hline 25 & 920821 & $20: 12: 46.7$ & 44.326 & 41.757 & & 1.9 & 99.4 & 9.07 & -0.32 & 3 \\
\hline
\end{tabular}

*Magnitude given in CAUCASUS NETWORK Information Product, Technical Reference Manual, IRIS/Joint Seismic Program Center (JSPC), Univ. Colorado, and Lamont-Doherty Earth Observatory, April 1994.

$†$ Magnitude given in PDE monthly list.

Distance $=$ mean epicentral distance for each event used in three-component analysis; score $=$ discriminant score from three-component analysis (see Fig. 7); $P g / L g=$ mean spectral ratio used in three-component analysis; $N=$ number of records used in three-component analysis.

$\left.-\alpha^{2} p^{2}\right)^{1 / 2}$ is imaginary. In this slowness range, there is a phase shift introduced between the vertical and radial components of the $S V$ contributions to the surface displacement that gives rise to elliptical polarization patterns (Kennett, 1991). Therefore, for the $P$ trace, the $Z$ component is Hilbert transformed before the transformation (7a) for slownesses greater than $1 / \alpha$.

The composite incident wave-vector traces show that indeed the $P$ waves are mainly on the $P$-wave-vector component, while $S$ waves ( $L g$ ) are dominantly on the $S V$-wavevector component (Fig. 9), hence the amplitudes of major crustal phases reveal clearer radiation characteristics from the source. In particular, the relatively small $S H$ in the regional $S$ waves clearly indicates the signal being generated by an explosion source. A very useful result of the freesurface correction is that we can make direct comparison between the $P_{-}, \mathrm{SV}$-, and $\mathrm{SH}$-wave amplitude in a particular group velocity window and so get closer to the radiation characteristics from the source. This is of potential significance for discriminating different types of sources, and we examine the usefulness of free-surface-corrected data in the following section.

\section{Network-Averaged Free-Surface-Corrected Three-Component $\mathrm{Pg} / \mathrm{Lg}$ Ratio}

We examined the $P g / L g$ spectral ratio method to discriminate earthquakes from explosions using the free-surface-corrected regional three-component records. The $P g / L g$ ratios from the free-surface-corrected $P, S V$, and $S H$ seismogram of three-component regional records are obtained for each station by defining

$$
\frac{P g}{L g}=\frac{P g_{P}}{\sqrt{L g_{S V}^{2}+L g_{S H}^{2}}}
$$

where $P g_{p}=$ spectral amplitude of the $P$ wave on the $P$ seismogram, $L g_{S V}=$ spectral amplitude of the $L g$ wave on the $S V$ seismogram, and $L g_{S H}=$ spectral amplitude of the $L g$ wave on the $S H$ seismogram. A single $P g / L g$ ratio is obtained for each station, and the network mean $P g / L g$ ratio is obtained by averaging over stations for each event. The network-averaged $\log _{10}(P g / L g)$ ratios based on equation (8) from $P, S V$, and $S H$ seismograms are plotted in Figure 10. The separation of the spectral ratios of earthquake and ex- 
Table 3

List of Quarry Blasts Used in This Study

\begin{tabular}{|c|c|c|c|c|c|c|c|}
\hline \multirow[b]{2}{*}{ Id \# } & \multicolumn{2}{|c|}{ Origin Time } & \multirow{2}{*}{$\begin{array}{c}m_{b} \\
\text { (JSPC) }\end{array}$} & \multirow{2}{*}{$\begin{array}{c}\text { Distance } \\
(\mathrm{km})\end{array}$} & \multicolumn{3}{|c|}{ Three-component Analysis } \\
\hline & (yr mo dy) & (hh:mm:sec) & & & Score & $P g / L g$ & $N$ \\
\hline \multicolumn{8}{|c|}{ Tyrnauz mine $\left(43.386^{\circ} \mathrm{N}, 42.871^{\circ} \mathrm{E}\right)$} \\
\hline 1 & 920223 & $07: 02: 43.4$ & 3.3 & 89.5 & -8.07 & 0.53 & 4 \\
\hline 2 & 920311 & $13: 39: 01.5$ & 3.2 & 96.8 & -15.10 & 0.64 & 3 \\
\hline 3 & 920322 & $07: 29: 38.7$ & & 86.2 & -2.09 & 0.34 & 2 \\
\hline 4 & 920329 & $06: 42: 46.5$ & 2.7 & 86.2 & -6.56 & 0.40 & 2 \\
\hline 5 & 020402 & $12: 19: 39.3$ & & 80.0 & -10.79 & 0.51 & 3 \\
\hline 6 & 920409 & $13: 02: 38.9$ & & 80.0 & -4.79 & 0.37 & 3 \\
\hline 7 & $920412^{*}$ & $08: 04: 34.1$ & & 80.0 & -7.66 & 0.44 & 3 \\
\hline 8 & 920419 & $03: 53: 37.0$ & & 79.7 & -8.53 & 0.46 & 3 \\
\hline 9 & 920423 & $13: 28: 42.4$ & 2.0 & 86.2 & -5.12 & 0.44 & 2 \\
\hline 10 & 920607 & $05: 39: 56.2$ & 1.5 & 65.6 & -7.02 & 0.35 & 2 \\
\hline 11 & 920607 & $05: 55: 40.7$ & 2.1 & 83.0 & -10.14 & 0.38 & 3 \\
\hline 12 & 920614 & $04: 37: 18.3$ & & 83.0 & -7.81 & 0.44 & 3 \\
\hline 13 & 920705 & $06: 35: 03.4$ & & 65.6 & -5.78 & 0.36 & 2 \\
\hline 14 & 920712 & $06: 35: 54.8$ & & 66.0 & -5.50 & 0.20 & 2 \\
\hline $15^{(\mathrm{a})}$ & $930822 *$ & $05: 09: 38.4$ & $18.9 \div$ & 53.4 & -11.41 & 0.35 & 3 \\
\hline $16^{(b)}$ & $930822 *$ & $06: 24: 24.4$ & $24.0 \div$ & 53.4 & -6.70 & 0.27 & 3 \\
\hline $17^{(\mathrm{c})}$ & $930829 *$ & $05: 23: 58.6$ & $10.2 \div$ & 53.4 & -12.52 & 0.29 & 3 \\
\hline \multicolumn{8}{|c|}{ Ust Djeguta $\left(44.10^{\circ} \mathrm{N}, 42.05^{\circ} \mathrm{E}\right)$} \\
\hline 18 & 920127 & $09: 32: 45.1$ & & 56.2 & -7.66 & 0.23 & 1 \\
\hline 19 & 920219 & $11: 34: 41.7$ & & 52.2 & -6.74 & 0.26 & 3 \\
\hline 20 & 920316 & $11: 37: 17.1$ & & 71.9 & -6.15 & 0.24 & 1 \\
\hline 21 & 920414 & $11: 02: 09.7$ & & 69.8 & -1.67 & 0.13 & 3 \\
\hline 22 & 920421 & $11: 15: 58.2$ & & 69.8 & -2.32 & 0.08 & 3 \\
\hline 23 & 920609 & $14: 03: 37.8$ & & 69.3 & -8.40 & 0.33 & 1 \\
\hline 24 & 920624 & $09: 41: 05.9$ & & 85.3 & -6.43 & 0.22 & 2 \\
\hline \multicolumn{8}{|c|}{ Zhako-Krasnogorskaya $\left(43.941^{\circ} \mathrm{N}, 41.831^{\circ} \mathrm{E}\right)$} \\
\hline 25 & $920508 *$ & $11: 23: 50.0$ & & 63.7 & -0.99 & 0.08 & 2 \\
\hline
\end{tabular}

*Locations and origin times provided by F. Riviére-Barbier, Center for Seismic Studies, Washington, D.C. (personal comm., March 1994)

$\dagger$ Metric tons (B. Stump, personal comm., May 1994)

(a) Underground $1-3$ shots.

(b) Surface ripple-fired.

(c) Undergound 1-3 shots.

Distance $=$ mean distance for each event used in three-component analysis. Other notation is the same as in Table 2 .

plosion groups appears to be similar to the three-component $\mathrm{Pg} / \mathrm{Lg}$ ratios (Fig. 6).

The sample data sets consisting of 25 earthquakes and 25 explosions were analyzed using the linear discriminant function as in the previous sections. For each event, network-averaged free-surface-corrected three-component $\log _{10}(P g / L g)$ ratios at frequencies of $8,10,12,14,16,18$, and $20 \mathrm{~Hz}$ correspond to the variables $r_{1}, r_{2}, r_{3}, r_{4}, r_{5}$, and $r_{6}$. The linear discriminant function that best separates the two types of source is

$$
\begin{aligned}
D(\mathbf{r})= & -5.09+7.17 r_{1}-17.43 r_{2} \\
& +17.52 r_{3}-32.52 r_{4}+11.59 r_{5}-12.15 r_{6},
\end{aligned}
$$

and the Mahalanobis $D$-squared measure of distance between the two population means is $\Delta^{2}=15.15$, higher than either of the two values obtained above. Assuming equal prior probabilities for the two groups, and applying the previous classification rule to the earthquake and explosion data, we find that two explosions are classified incorrectly and that the total misclassification probability is very low, namely $2.6 \%$. The two misclassified explosions are events 21 and 25 (Table 3 ).

The discriminant score for each event is plotted in Figure 11 with respect to the network mean free-surface-corrected $\log _{10}(P g / L g)$ spectral amplitude ratio of each event. It is shown in Figure 11 that the earthquake records from various paths in southern Russia have a mean free-surfacecorrected three-component $P g / L g$ spectral ratio of about 0.32 in the frequencies 8 to $18 \mathrm{~Hz}$, while the explosion records show a mean of about 1.0. These mean $P g / L g$ ratios are lower than the vertical-component or three-component data discussed earlier (see, e.g., Figs. 5 and 7) by about a factor 2 to 3 , and there is a clearer separation of $P g / L g$ spectral ratios of the earthquake and explosion populations. Notice that two distant earthquakes, numbers 2 and 16, have higher $P g / L g$ ratios than other earthquakes (Fig. 11). The free-surface-corrected $P g / L g$ spectral ratios at frequencies 5 


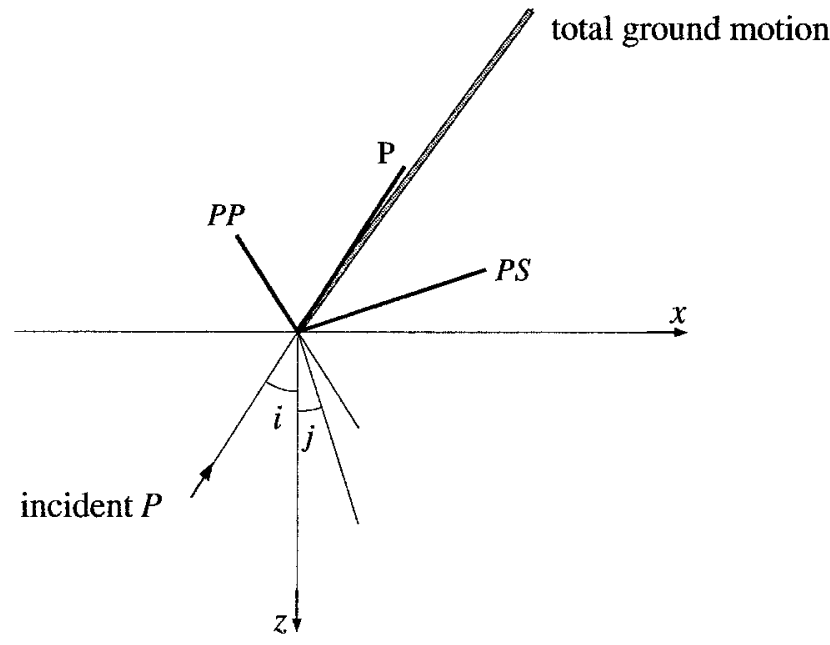

Figure 8. Sketch of ground motions in a homogeneous half-space for a $P$-wave incident upon a free surface with unit amplitude and an angle of incidence $i \approx 33.37^{\circ}(=0.1 \mathrm{sec} / \mathrm{km})$. Surface $P$ and $S$ velocities are $\alpha=5.5 \mathrm{~km} / \mathrm{sec}$ and $\beta=3.1 \mathrm{~km} / \mathrm{sec}$, respectively. The total ground motion of the free surface (shaded line) itself is shown above the surface level $z=0$ and has amplitude 2.03 times the incident wave with an apparent angle of incidence $i^{\prime}=36.12^{\circ}$. Scattered waves $P P$ and $P S$, which are downgoing waves, are plotted above the level $z=0$, indicating the directions of true particle motion. The amplitudes of particle motions are indicated by their length.

to $20 \mathrm{~Hz}$ indicate that for the earthquakes, the radiated seismic amplitudes are partitioned into $P$ and $S V$-SH waves with a ratio 1:3, while for the explosions, $P$ and $S V$ waves have comparable amplitudes. Use of the free-surface-corrected $P g / L g$ spectral ratio improves the discrimination power of high-frequency regional data by about $4.5 \%$ over the use of single vertical-component ratios (92.9\% versus $97.4 \%$ ).

\section{Correction of Distance Effects on the $P g / L g$ Spectral Amplitude Ratio}

The sample data- 25 earthquakes and 25 explosionsused for the linear discriminant function analyses in the previous sections come from unevenly distributed sources. The explosions were exclusively from three mining areas, while the earthquakes were widely distributed in the region (Fig. 3 ). For simplicity, we could have chosen only earthquakes that were located at distance ranges comparable to the explosions; however, we opted to select earthquakes at various locations to sample a representative seismicity of the region, for developing seismic discriminants for practical monitoring purposes in the region. Because the distribution of the earthquakes and explosions used were uneven, we examined the effects of distance on the spectral ratio.

The vertical-component $P g / L g$ ratios of 82 earthquake records and 78 explosion records are plotted against distance in Figure 12. The mean of the $P g / L g$ ratios in the frequency

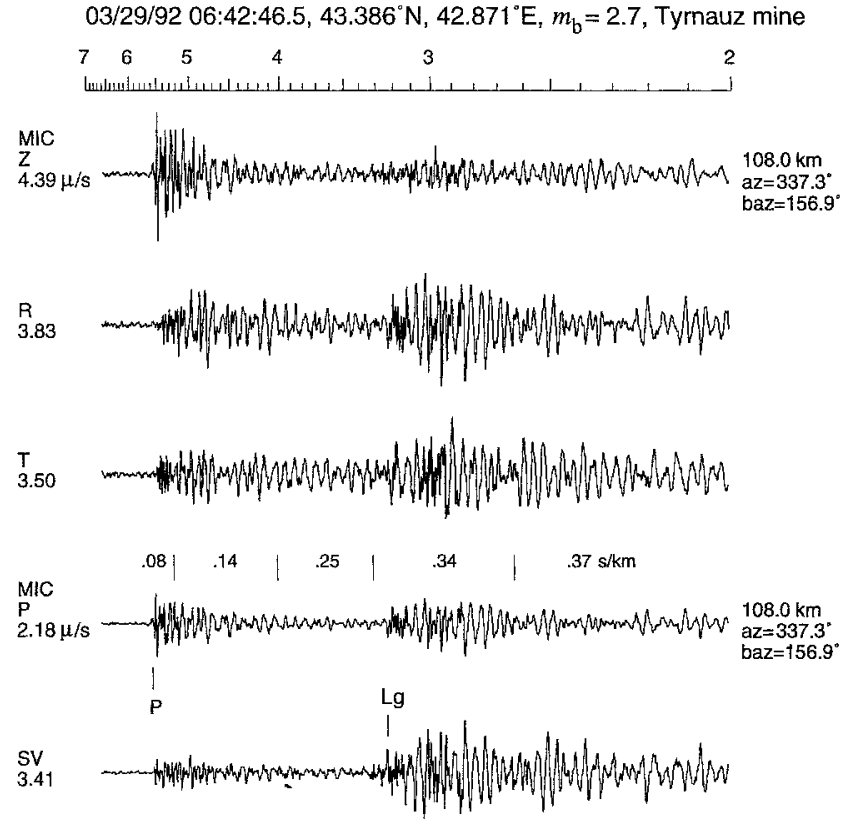

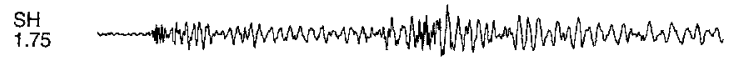

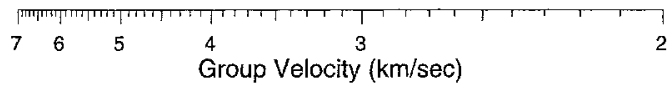

Figure 9. (Upper three traces) Rotated three-component records from an explosion in Tyrnauz mine ( $Z$, $R$, and $T$ ). The $P$ waves are on the $Z$ component, while $S$ waves are dominantly on the $R$ and $T$ components. (Lower three traces) Composite incident wave-vector component traces $(P, S V$, and $S H)$ produced by applying free-surface correction operators successively along the trace. The breaks between different slowness bands are marked by vertical bars, and the slowness (in sec/km) used in each group velocity window is indicated. All traces are plotted with the same amplitude scale. Trace peak-to-peak amplitudes in $\mu / \mathrm{sec}$ are given in each trace.

band 8 to $18 \mathrm{~Hz}$ are plotted, and an average trend, calculated from each of the distance bins in every $20-\mathrm{km}$ interval, is superposed in the figure. The earthquake data show a weak distance dependence in the ratio, caused probably by differences in attenuation of $P g$ (or $P$ ) and $L g$ (or $S$ ) phases over distance. The $P g / L g$ ratios at distances less than about 100 $\mathrm{km}$ are about -0.1 logarithmic units (1.u.), and they increase to 0.2 l.u. at distance ranges of 100 to $180 \mathrm{~km}$. The ratios reach to 0.4 l.u. at distance ranges of 180 to $240 \mathrm{~km}$ (Fig. 12). Hence, the $P g / L g$ ratios of earthquake data increase by a factor of about 3 within the distance range of 80 to 200 $\mathrm{km}$. We notice a spectral ratio minimum in the earthquake data at about $170 \mathrm{~km}$. It is probably associated with the crossover distance in $P$-wave travel-time curve where $P n$ phase becomes the first arrival phase. It represents a change in the regional wave field in which $P$ waves start leaking to 


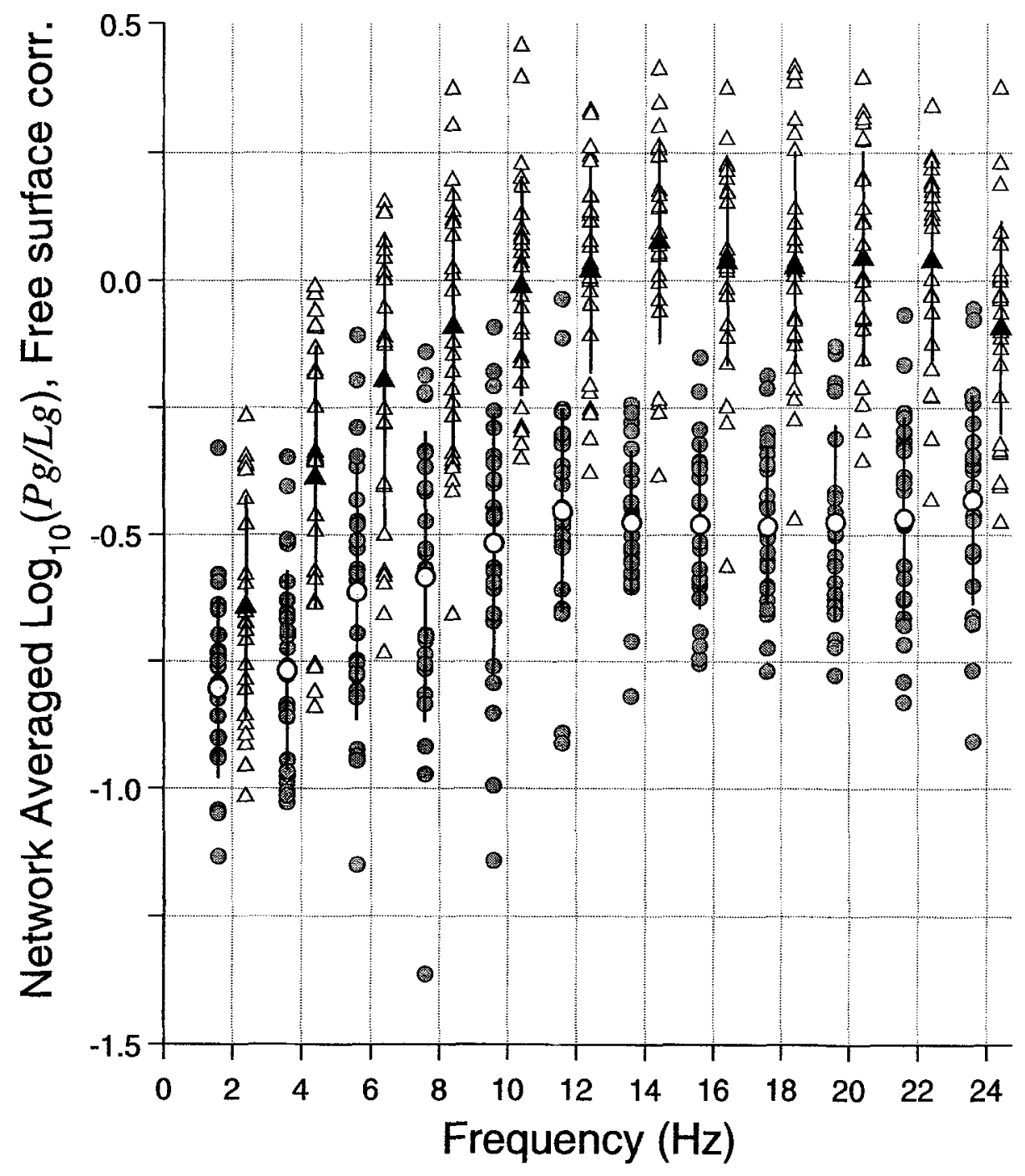

Figure 10. Network-averaged $\log _{10}(P g / L g)$ spectral amplitude ratios at 12 discrete frequency points, from the free-surface-corrected three-component records $(P, S V$, and $S H$ seismogram) used in discrimination analysis, are plotted for earthquakes (shaded circles) and explosions (open triangles). Other notation is the same as in Figure 4.

the upper mantle, thereby reducing $P$ energy compared to $S$ or $L g$. This spectral ratio minimum occurs at approximately $180 \mathrm{~km}$ for the northeastern U.S. data set studied by Kim et al. (1993). It is noted that the "distance" here does not represent a simple length scale, rather it is the "path length" within a complex 3D earth structure, since the paths from most of the distant earthquakes cross a major tectonic boundary-Greater Caucasus (see Figs. 1 and 3). Nevertheless, we examine a first-order approximation to account for the effects of "distance" on the spectral ratio.

For $P g$ and $L g$ phases, the spectral amplitude decay with distance can be written in the form

$$
A(\Delta, f)=A_{0}(f) \exp (-\gamma(f) \Delta) \Delta^{-\kappa}
$$

which consists of an anelastic attenuation factor and the geometrical spreading factor, where $\Delta=$ epicentral distance in kilometers, $A(\Delta, f)=$ observed spectrum at distance $\Delta$, $A_{0}(f)=$ source spectrum, $\gamma(f)=$ anelastic attenuation coefficient, and $\kappa=$ exponent for geometrical spreading. $\gamma(f)$ is related to quality factor $Q(f)$ by the formula $\gamma(f)=\pi f I$ $[Q(f) U]$, where $U$ is the group velocity. $Q(f)$ is a function of frequency, $f$, and is assumed to have the form $Q(f)=$ $Q_{0} f^{\eta}$, where $Q_{0}$ is the quality factor at a reference frequency $(f=1 \mathrm{~Hz})$ and $\eta$ is the exponent of frequency. Using the differences, $\bar{\gamma}(f)=\gamma_{P}(f)-\gamma_{S}(f)$ and $\bar{\kappa}=\kappa_{P}-\kappa_{S}$, the distance-corrected spectral ratio, $\log _{10}\left\{P g_{0}(f) / L g_{0}(f)\right\}$, can be written as

$$
\begin{aligned}
\log _{10}\{ & \left.P g_{0}(f) / L g_{0}(f)\right\} \\
\quad & =\log _{10}\left[\{P g(f, \Delta) / L g(f, \Delta)\} \exp (\bar{\gamma}(f) \Delta) \Delta^{\bar{F}}\right] .
\end{aligned}
$$

A recent study by Abers and Sarker (1996) for the northern Caucasus region suggests average frequency-indepen- 


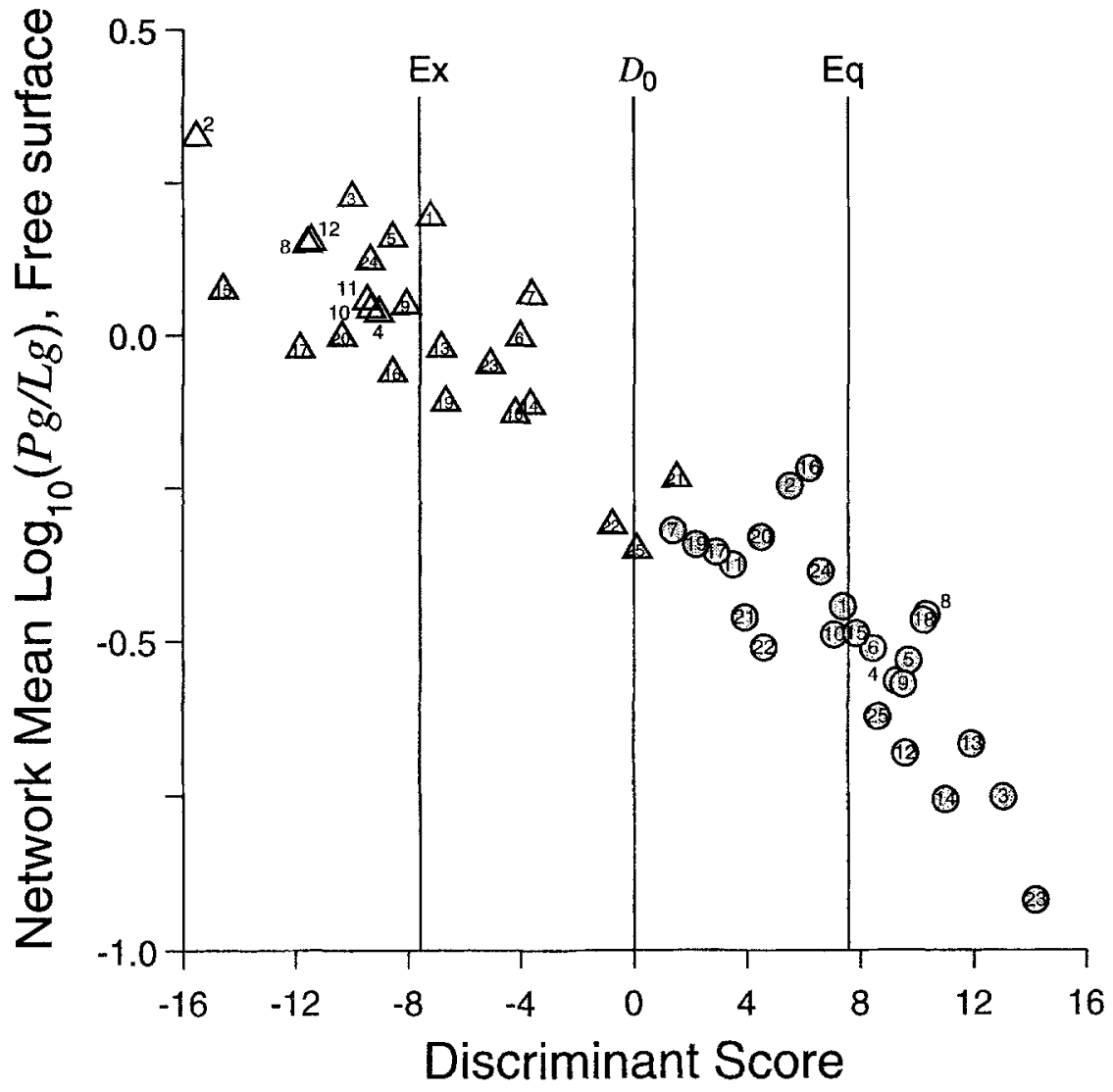

Figure 11. Discriminant scores of earthquakes (shaded circles) and explosions (triangles) of the sample data from the free-surface-corrected three-component records are plotted with their mean network-averaged $\log _{10}(P g / L g)$ ratios. Other notation is the same as in Figure 5.

dent $Q$ values of $1070 \pm 200$ and $2130 \pm 600$ for $P g$ (or $P$ ) waves in the predominantly mountain and shield path, respectively. They obtained $Q$ values of $775 \pm 75$ and 2060 \pm 315 for $L g$ (or $S$ ) waves in the mountain and shield path, respectively, in the frequencies up to $10 \mathrm{~Hz}$. The boundary between the two tectonic features (i.e., mountain and shield) lies approximately at the northern edge of the Greater Caucasus (Fig. 1), and it closely follows the northern edge of the 2000-m contour line shown in Figure 3. These $Q$ values for $L g$ waves with $U=3.4 \mathrm{~km} / \mathrm{sec}$ give $\gamma(1 \mathrm{~Hz})=0.0012$ $\mathrm{km}^{-1}$ and $0.00045 \mathrm{~km}^{-1}$ for mountain and shield path, respectively. These attenuation coefficients seem to be somewhat small when compared with other more stable regions. For instance, a recent study by Shi et al. (1996) indicates an average $Q_{0} \approx 625$ and $\eta \approx 0.25$ for $L g$ waves in the northeastern United States, which give $\gamma(1 \mathrm{~Hz})=0.00148 \mathrm{~km}^{-1}$ at a reference frequency of $1 \mathrm{~Hz}$ with $U=3.4 \mathrm{~km} / \mathrm{sec}$. For $P g$ waves, above $Q$ values with $U=6 \mathrm{~km} / \mathrm{sec}$ give $\gamma(1 \mathrm{~Hz})$ $=0.00049 \mathrm{~km}^{-1}$ and $0.00025 \mathrm{~km}^{-1}$ for mountain and shield path, respectively.

We assume that the geometrical spreading for both $P$ and $S$ waves at short distances $(\Delta \leqq 100 \mathrm{~km})$ is similar and that it may be approximated by $\Delta^{-1}$. The geometrical spreading for spectral amplitude of $L g$ waves ranges from
$\Delta^{-0.5}$ to $\Delta^{-1}$ (e.g., Campillo et al., 1984), while $P g$ waves range between $\Delta^{-1}$ and $\Delta^{-1.5}$ (e.g., Langston, 1982) at regional distances. These values indicate a differential geometrical spreading factor with an exponent of $\bar{\kappa}=0$ for $\Delta$ $\leqq 100 \mathrm{~km}$ and $\bar{\kappa} \approx 0.5$ for $\Delta>100 \mathrm{~km}$.

Combining the above differential geometrical spreading and attenuation coefficient between $P g$ and $L g$ waves, the distance correction for the spectral ratio in the northern $\mathrm{Cau}-$ casus region can be written as $\exp \left(-0.0002 \mathrm{~km}^{-1} f \Delta\right) \Delta^{\bar{\epsilon}}$ and $\exp \left(-0.0007 \mathrm{~km}^{-1} f \Delta\right) \Delta^{\check{\kappa}}$ for shield and mountain path, respectively. These distance correction factors are plotted in Figure 13 against distance. We indicate as "mixed path" the propagation paths consisting of shield and mountain paths in about equal parts.

These distance corrections are applied to the verticalcomponent spectral ratios for each path, and resulting ratios are plotted in Figure 12 against epicentral distance for comparison. As expected from Figure 13, the corrected ratios in the high-frequency band ( 8 to $18 \mathrm{~Hz}$ ) are about 0.1 l.u. lower for earthquake records at a distance of $100 \mathrm{~km}$, while the corrected ratios are about 0.4 1.u. lower than the raw uncorrected ratios at a distance of $240 \mathrm{~km}$ (Fig. 12). The trend after the correction shows a much weaker distance dependence of the ratios and suggests that the distance correction 


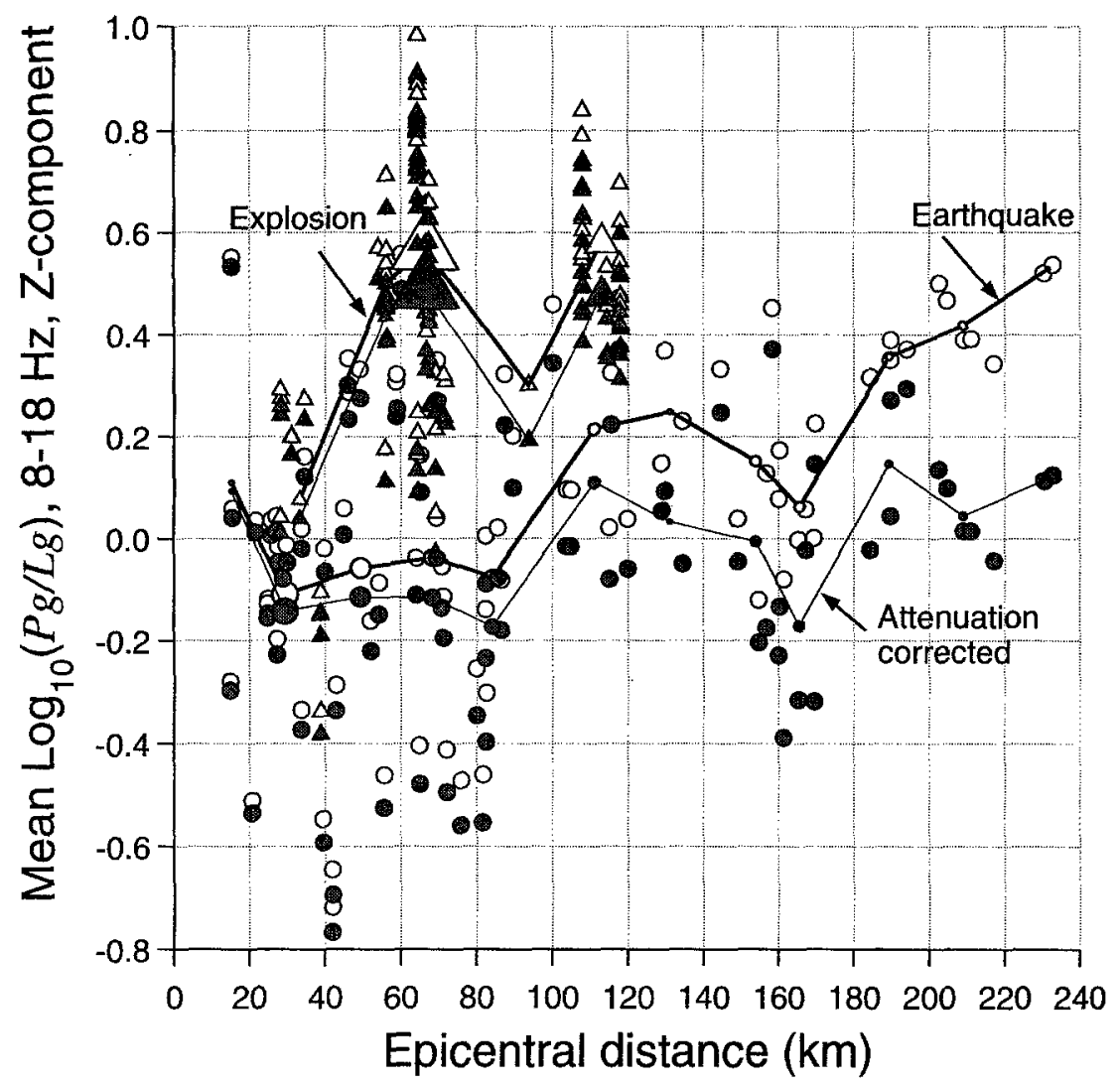

Figure 12. The vertical-component mean high-frequency (8 to $18 \mathrm{~Hz}) P g / L g$ ratios of 82 earthquake records (open circles) and 78 explosion records (open triangles) are plotted against distance. Corresponding distance-corrected spectral ratios are plotted with shaded symbols. An average trend, calculated from each of the $20-\mathrm{km}$-distance bins, is superposed (thick lines), and an average trend after the distance correction is plotted for comparison (thin lines). The mean ratio in each distance bin is plotted at the mean distance in each bin for earthquake (open circle) and for explosion (open triangle), and their sizes are proportional to the density of the bin.

using equation (11) may be a reasonable first-order approximation to account for distance effects on the spectral ratio.

\section{Distance-Corrected Network-Averaged \\ Vertical-Component $P g / L g$ Ratio}

We examined the $P g / L g$ spectral ratio method to discriminate earthquakes from explosions using distance-corrected regional vertical-component records. The $P g / L g$ ratios from vertical-component records corrected for distance by using equation (11) are used as the sample data set. A single $P g / L g$ ratio is obtained for each station, and the network mean $P g / L g$ ratio is obtained by averaging over stations for each event. The network-averaged $\log _{10}(P g / L g)$ ratios corrected for the distance are plotted in Figure 14. The mean and standard deviation at each frequency is superposed in the figure to indicate the quality of the distance-corrected ratios. The distance-corrected spectral ratios show smaller $\mathrm{Pg} / \mathrm{Lg}$ values and smaller scatter than the uncorrected ratios at all frequencies up to about $20 \mathrm{~Hz}$. The separation of the spectral ratios of earthquake and explosion groups appears to be similar to the uncorrected ratios at low frequencies $(<8$
$\mathrm{Hz}$ ), but the separation between the two groups is increasing with frequency for frequencies greater than $10 \mathrm{~Hz}$. This is due to the fact that several earthquakes were more distant than explosions.

For frequencies higher than $20 \mathrm{~Hz}$, corrected ratios show greater scatter than the raw ratios, suggesting that the distance correction applied was not appropriate at these high frequencies (Fig. 14). This is probably due to the fact that the $Q$ factors reported by Abers and Sarker (1996) are obtained for frequencies up to $10 \mathrm{~Hz}$. The frequency-independent $Q$ factors we have used may not be applicable at frequencies higher than $20 \mathrm{~Hz}$ as shown by Shi et al. (1996) who showed the necessity for frequency-dependent $Q$ in regional waves studied up to $35 \mathrm{~Hz}$. However, we expect that the frequency-independent $Q$ we have used in the present study provides fairly reliable spectral ratios for frequencies up to $18 \mathrm{~Hz}$, as shown in Figure 14, and a sampled version will be used in the following discrimination analysis.

The sample data sets consisting of 25 earthquakes and 25 explosions (vertical component only) were analyzed using the linear discriminant function as in the previous sec- 


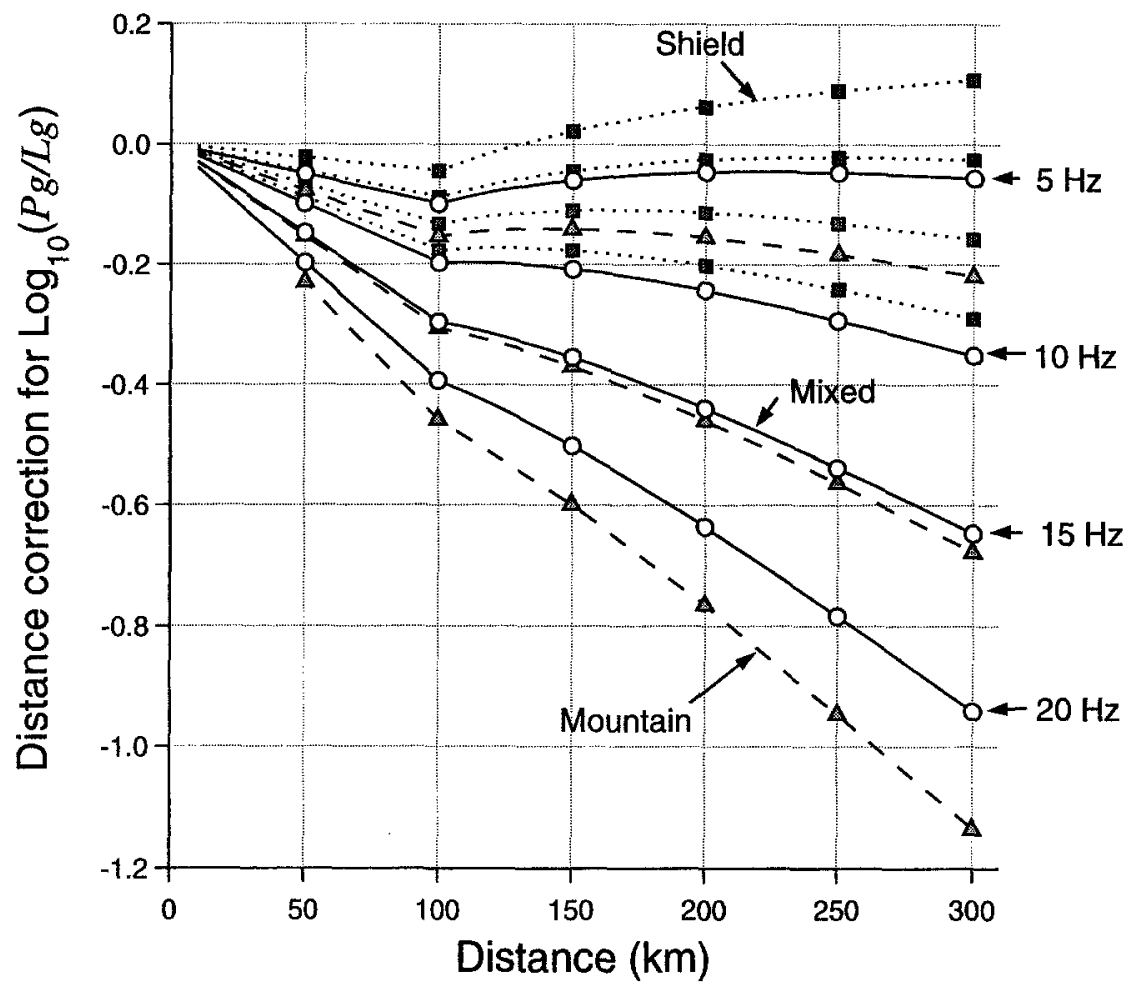

Figure 13. Distance correction factors for $\log _{10}(P g / L g)$ spectral ratios for the northern Caucasus region are plotted as a function of frequencies and distances. Correction factors for various paths are indicated: shield path (dotted lines), mountain (dashed lines), and mixed path--path crossing both regions (solid lines). The correction factor includes a differential geometrical spreading of $\Delta^{0.5}$ (for $\Delta>100 \mathrm{~km}$ ) and attenuation coefficient $\left(\gamma_{P}-\gamma_{S}\right)$.

tions. For each event, distance-corrected network-averaged $\log _{10}(P g / L g)$ ratios at discrete frequencies, 8 to $18 \mathrm{~Hz}$, are used as before. The linear discriminant function that best separates the two types of source is

$$
\begin{array}{r}
D(r)=6.06+16.67 r_{1}-15.57 r_{2}-9.46 r_{3} \\
-18.33 r_{4}+4.36 r_{5}-6.93 r_{6},
\end{array}
$$

and the Mahalanobis $D$-squared measure of distance between the two population means is $\Delta^{2}=14.14$, much higher than the value obtained for the distance-uncorrected case (cf. $\Delta^{2}=8.67$ ). Assuming equal prior probabilities for the two groups, and applying the previous classification rule to the earthquake and explosion data, we find that an earthquake is classified incorrectly and that the total misclassification probability is $3 \%$. This is very close to the misclassification probability obtained for the three-component analysis. Records from a single misclassified earthquake (event 16) show much stronger $P g$ than $L g$ signals on vertical components. As such, the event could only be correctly classified by using three-component data.

By examining a variety of different discrete frequency bands using linear discriminant function analysis, we found that the frequency band 6 to $14 \mathrm{~Hz}$ provided the best dis- crimination power for this data. Apparently, the distance correction has increased the discrimination power for the frequency band 8 to $14 \mathrm{~Hz}$, while reducing the discrimination power for higher-frequency band 16 to $18 \mathrm{~Hz}$.

The discriminant score for each event is plotted in Figure 15 with respect to the network mean distance-corrected $\log _{10}(P g / L g)$ spectral ratio of each event. The mean $P g / L g$ ratios are lower than the distance-uncorrected vertical component (see, e.g., Fig. 5), and there is a clearer separation of $\mathrm{Pg} / \mathrm{Lg}$ spectral ratios of the earthquake and explosion populations than the uncorrected case (cf. Figs. 5 and 15). This preliminary analysis indicates that use of the distance-corrected $P g / L g$ spectral ratio improves the discrimination power of high-frequency regional data by about $4.1 \%$ over the use of single vertical-component ratios $(92.9 \%$ versus $97.0 \%$ ). However, more accurate information regarding the geometrical spreading and anelastic attenuation of the regional phases such as $P g$ and $L g$ are required for applying the distance correction to $P g / L g$ ratios with confidence.

\section{Discussion and Conclusions}

We find that $P g$ waves and $L g$ waves from earthquakes have comparable high-frequency content over the broad, high-frequency band width of 5 to $20 \mathrm{~Hz}$ at regional dis- 


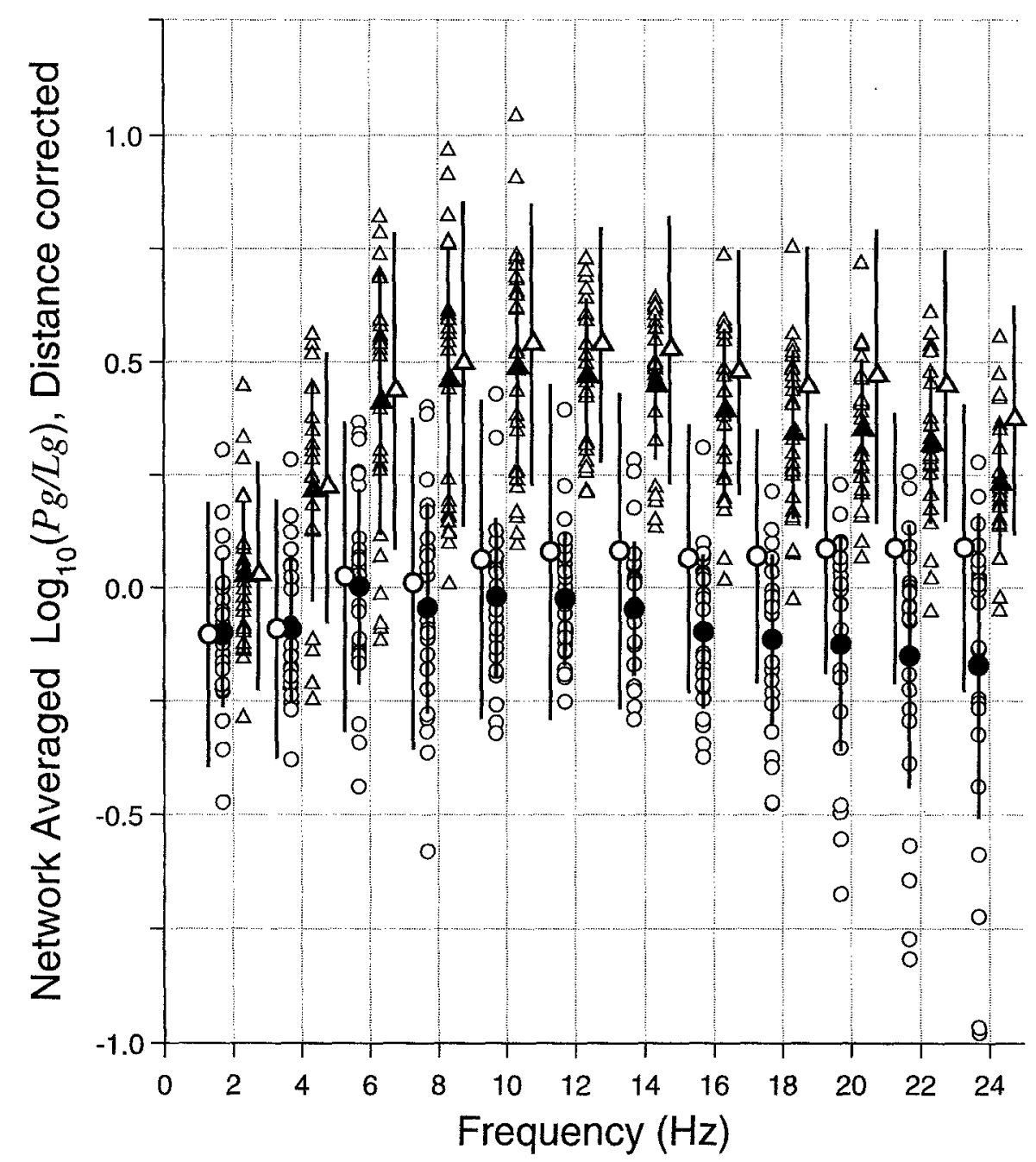

Figure 14. Network-averaged vertical-component $\log _{10}(P g / L g)$ spectral ratios corrected for the distance are plotted for earthquakes (shaded circles) and explosions (open triangles). At each discrete frequency, mean values of distance-uncorrected ratios are also plotted for earthquakes (open circles) and explosions (solid triangles) for comparison. Other notation is the same as in Figure 4.

tances in southern Russia near Kislovodsk: The mean vertical-component $\mathrm{Pg} / \mathrm{Lg}$ ratio for 82 earthquake records is about 1.3. For explosions (multiple-hole instantaneous and ripple-fired explosions), the $P g$ waves are much stronger than $L g$ : The mean vertical-component $P g / L g$ ratio for 78 explosion records in the same frequency band is about 3.2. These mean $\mathrm{Pg} / \mathrm{Lg}$ ratios, 1.3 for earthquakes and 3.2 for explosions, are much higher than the ratios observed in tectonically stable eastern United States, where the ratios are 0.5 and 1.25 for the earthquakes and explosions, respectively (Kim et al., 1993).

The $P g / L g$ spectral amplitude ratio using vertical-component records in a broad high-frequency band of 4 to 18 $\mathrm{Hz}$ provides discrimination power with a total misclassification probability of about 6.3 to $7.1 \%$. In a lower-frequency band, 2 to $10 \mathrm{~Hz}, P g / L g$ ratios show poor results, and the separation between explosions and earthquakes is less clear with a total misclassification probability of about $14.3 \%$ (Table 4). However, when a single $P g / L g$ ratio at $12 \mathrm{~Hz}$ is added in the low-frequency band, the new frequency band, 2 to 12 $\mathrm{Hz}$, improves the discrimination power by up to about $6 \%$ for vertical- as well as three-component data (Table 4). This significant improvement, which is due to the addition of a single $12-\mathrm{Hz} P g / L g$ ratio, illustrates the importance of highfrequency data in regional seismic discrimination.

The $P g / L g$ spectral ratios using three-component regional records, corrected for the free-surface effect, improve the discrimination power of the spectral ratio method. In the frequency band 8 to $18 \mathrm{~Hz}$, the free-surface-corrected, threecomponent $P g / L g$ spectral ratio provides discrimination power with a total misclassification probability of only $2.6 \%$.

In the case that free-surface effects cannot be corrected with confidence, then the $P g / L g$ spectral ratios of rotated, three-component, regional records may be used as a discrim- 


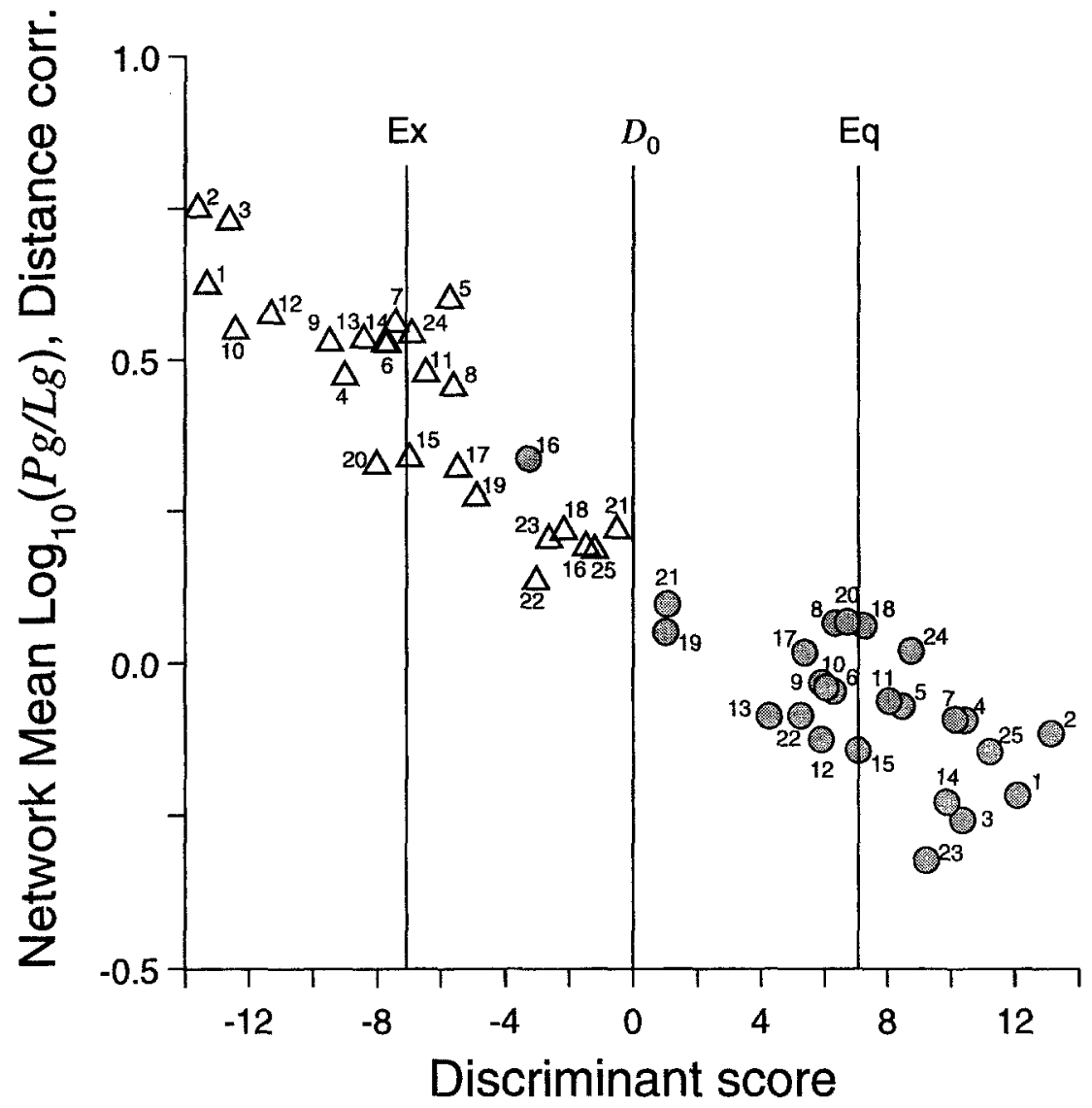

Figure 15. Discriminant scores of earthquakes (shaded circles) and explosions (open triangles) of the distance-corrected vertical-component data are plotted with their mean network-averaged $\log _{10}(P g / L g)$ ratios. Note that these two populations are also well separated by mean $\log _{10}(P g / L g)$ ratios. One earthquake is incorrectly classified, and the total misclassification probability is $3.0 \%$. Each event is identified by its ID number given in Tables 2 and 3. Other notation is the same as in Figure 5.

Table 4

Comparison between Different Frequency Bands in Application of the $P g / L g$ Discriminant

\begin{tabular}{|c|c|c|c|c|c|c|c|c|}
\hline \multirow[b]{2}{*}{ Case } & \multicolumn{2}{|c|}{$2-10 \mathrm{~Hz}$} & \multicolumn{2}{|c|}{$10-18 \mathrm{~Hz}$} & \multicolumn{2}{|c|}{$2-12 \mathrm{~Hz}$} & \multicolumn{2}{|c|}{$8-18 \mathrm{~Hz}$} \\
\hline & $\begin{array}{c}P \\
(\%)\end{array}$ & $\Delta^{2 \dagger}$ & $\begin{array}{c}P \\
(\%)\end{array}$ & $\Delta^{2}$ & $\begin{array}{c}P \\
(\%)\end{array}$ & $\Delta^{2}$ & $\begin{array}{c}P \\
(\%)\end{array}$ & $\Delta^{2}$ \\
\hline Vertical component & 85.70 & 4.5 & 92.10 & 8.0 & 91.56 & 7.6 & 92.95 & 8.7 \\
\hline Three component & 87.96 & 5.5 & 96.96 & 14.1 & 90.29 & 6.7 & 96.96 & 14.1 \\
\hline Free-surface corrected & 89.85 & 6.5 & 97.14 & 14.5 & 93.01 & 8.7 & 97.42 & 15.1 \\
\hline Distance corrected & 89.02 & 6.0 & 96.01 & 12.3 & 96.50 & 13.1 & 96.99 & 14.1 \\
\hline
\end{tabular}

*Probability.

$\dagger$ Mahalanobis $D$-squared measure.

inant. The use of rotated, three-component records improves the discrimination power over the vertical component. In the frequency band of 8 to $18 \mathrm{~Hz}$, the three-component $P g / L g$ spectral ratio provides discrimination power with a total misclassification probability of about $3 \%$.

The area around the Caucasus Network was not an ideal region to make free-surface corrections, because of site effects associated with low-velocity near-surface layering. Ap- plication of the free-surface correction to data acquired at a good hard-rock site gives a better separation of $P, S V$, and $S H$ signals on different traces. For such sites, we would therefore expect an even better discrimination performance for the free-surface-corrected $\mathrm{Pg} / \mathrm{Lg}$ ratio.

Preliminary analysis of applying distance corrections to the vertical-component $P g / L g$ spectral ratio indicates that use of the distance-corrected spectral ratios improves the 
discrimination power of high-frequency regional data by about $4 \%$ over the use of vertical-component ratios $(92.9 \%$ versus $97.0 \%$ ). However; accurate information regarding the geometrical spreading and anelastic attenuation of the regional phases, such as $P g$ and $L g$ at high frequency, are required for applying distance corrections with confidence for the region in question. Lacking more detailed information on amplitude decay of regional phases with distance, in particular frequency-dependent $Q$ factors for $P$ (or $P g$ ) and $L g$ waves, in the northern Caucasus region, we applied distance corrections to only the vertical-component $P g / L g$ ratios in this study.

We have shown that the $P g / L g$ ratio is an adequate discriminant for explosions from earthquakes (magnitude smaller than 4.5) in southern Russia and that the discrimination is improved by the use of three-component data together with a free-surface correction. The stability of the $\mathrm{Pg} / \mathrm{Lg}$ ratio in discriminating multiple-hole, ripple-fired explosions from small regional earthquakes is significant for seismic monitoring in southern Russia. Our results demonstrate the importance of data at frequencies up to at least $20 \mathrm{~Hz}$.

One basis for the empirical success of our proposed high-frequency $P g / L g$ ratio method is that, if selected spectral amplitude reinforcement occurs at the source (as is the case for ripple-fired blasts), it will affect both early $P$ phases as well as later-arriving $L g$ phases. Such spectral scalloping will be largely canceled in the ratio we have used. The ratio is a robust discriminant to the extent that there are cancellation of other characteristics, such as event size, corner frequency, some effects of focal depth, and instrument response.

The problem of certain earthquake radiation patterns is also minimized by using the network-averaged ratios and three-component records, for example, a strong $P g$ excitation but weak $L g$ excitation from a near-vertical strike-slip earthquake (see, e.g., Bouchon, 1981; Kim, 1987) that may diminish the discrimination power of the $P g / L g$ ratio method at some station azimuths, as indicated by Lilwall (1988).

A key to the portability of the $P g / L g$ spectral ratio discriminant to various parts of the world is the correction of regional signals for their source-receiver path effects. We have shown that the free-surface effect can be easily removed from the regional records and that free-surface-corrected, $P g / L g$ ratios using three-component records improve the discrimination power of the $P g / L g$ ratio method. Further, the dependence of the frequency content of $P$ and $S$ waves on specific propagation paths and local structure at the receiver is reduced by using the network-averaged $P g / L g$ ratio (see also Kim et al., 1993).

The $P g / L g$ spectral ratios may be taken for different frequencies, for instance, $P g$ (high-frequency) $/ L g$ (low-frequency) ratios or ratios at different frequencies within the same phase as $L g$ (high-frequency) $/ L g$ (low-frequency) ratios. Such cross-spectral measures may be attractive for discriminating between distant earthquakes and explosions, from a few hundred to a couple of thousand kilometers. However, we need to correct for instrument responses; and propagation path effects-notably the frequency-dependent anelastic attenuation-must be taken into account for the cross-spectral ratios to be meaningful. The multivariate analysis employed in this study implicitly takes such a crossspectral measure when a broad enough frequency band is chosen for the input variables.

It has been reported by some researchers (e.g., Fisk, 1993; Taylor, 1996) as well as in this study that the distance corrections-primarily for anelastic attenuation along the paths-applied for both the $P$ and $S$ signals enhance the discriminant power of $P g / L g$ ratios. However, we believe that it is difficult to correct signals for the effects of distance with confidence, unless special studies are carried out, for example, with empirical Green's functions. The $P g / L g$ spectral amplitude ratio method we propose is sufficiently reliable and robust that it can be used in discriminating chemical explosions (especially numerous mining and quarry blasts) from small regional earthquakes in the routine analysis of data from regional earthquake-monitoring networks.

In the case of regions with high attenuation such as Nevada, the $P g / L g$ spectral ratio method may be applicable with somewhat diminished discrimination power when compared with stable regions, since the frequency band available for regional signals in such a region may be limited to up to about $10 \mathrm{~Hz}$. A comparison of performance between lowand high-frequency band $P g / L g$ spectral ratios summarized in Table 4 indicates that the method should work in such regions (using frequencies up to $10 \mathrm{~Hz}$ ) with a total misclassification probability of about 10 to $15 \%$. It may be emphasized that although we have presented results of frequency bands with the best discrimination power for four different cases, any combination of frequency bands across the range of 2 to $18 \mathrm{~Hz}$ provides fairly good results (Table 4). For instance, for vertical-component data, any six discrete frequencies between 2 and $18 \mathrm{~Hz}$ provide a total misclassification probability of between 7.1 and $8.4 \%$ (Table 4).

\section{Acknowledgments}

G. Abers, D. Johnson, D. Lentrichia, R. Such, of the Lamont-Doherty Earth Observatory, and Igor Chernoby, of the Experimental Methodological Expedition, Institute of Physics of the Earth, Obninsk, Russia, put considerable effort into maintaining the Caucasus Network and assisted in collecting the data used in this study. Drs. Vitaly Khalturin and Florence Riviére-Barbier provided us with locations of known quarries in southern Russia. We thank Danny Harvey at JSPC for production of the catalog used here. Drs. B. Stump, W. Menke, and R. Shumway and an anonymous reviewer provided useful comments. This project was supported by the IRIS Subaward \#214 (under NSF Cooperative Agreement No. EAR-9023505) and by the Air Force Office of Scientific Research under Grant F49620-951-0026. Lamont-Doherty Earth Observatory contribution number 5621.

\section{References}

Abers, G. A. and G. Sarker (1996). Seismic source and structure in Iran from Joint Seismic Program array data: attenuation variations at the 
northern margin of Eurasian mountains, in Proc. of the 18th Annual Seismic Research Symposium on Monitoring a Comprehensive Test Ban Treaty, 4-6 September 1996, J. F. Lewkowicz, J. M. McPhetres, and D. T. Reiter (Editors), PL-TR-96-2153, ERP, No. 1195, Hanscom AFB, Massachusetts, July 1996, 1-9.

Aki, K. and P. G. Richards (1980). Quantitative Seismology: Theory and Methods, vol. 1, W. H. Freeman, New York.

Bennett, T. J. and J. R. Murphy (1986). Analysis of seismic discrimination capabilities using regional data from western United States events, Bull. Seism. Soc. Am. 76, 1069-1086.

Bouchon, M. (1981). A simple method to calculate Green's functions for elastic layered media, Bull. Seism. Soc. Am. 71, 959-971.

Campillo, M., M. Bouchon, and B. Massinon (1984). Theoretical study of the excitation, spectral characteristics, and geometrical spreading of regional seismic phases, Bull. Seism. Soc. Am. 74, 79-90.

Chael, E. P. (1988). Spectral discrimination of NTS explosions and earthquakes in the southwestern United States using high-frequency regional data, Geophys. Res. Lett. 15, 625-628.

Davis, J. C. (1986). Statistics and Data Analysis in Geology, 2nd ed., Wiley, New York, 646 pp.

Dowla, F. U., S. R. Taylor, and R. W. Anderson (1990). Seismic discrimination with artificial neural networks: preliminary results with regional spectral data, Bull. Seism. Soc. Am. 80, 1346-1373.

Dysart, P. S. and J. J. Pulli (1990). Regional seismic event classification at the NORESS array: seismological measurements and the use of trained neural networks, Bull. Seism. Soc. Am. 80, 1910-1933.

Evernden, J. F., C. B. Archambeau, and E. Cranswick (1986). An evaluation of seismic decoupling and underground nuclear test monitoring using high-frequency seismic data, Rev. Geophys. 24, 143-215.

Fisher, R. A. (1936). The use of multiple measurement in taxonomic problems, Ann. Eugen. 7, 179-188.

Fisk, M. D. (1993). Event identification analysis of the 31 December 1992 Novaya Zemlya event with attenuation corrections, Mission Research Corporation, MRC-R-1459, 48 pp.

House, L. and J. Boatwright (1980). Investigation of two high stress drop earthquakes in the Shumagin seismic gap, Alaska, J. Geophys. Res. 85, 7151-7165.

Jebe, E. H. and D. E. Willis (1964). An application of the discriminant function technique to seismic records, Earthquake Notes 35, 28-49.

Kadinsky-Cade, K., M. Barazangi, J. Oliver, and B. Isacks (1981). Lateral variations of high-frequency seismic wave propagation at regional distances across the Turkish and Iranian Plateaus, J. Geophys. Res. 86, 9377-9396.

Kennett, B. L. N. (1991). The removal of free surface interactions from three-component seismograms, Geophys. J. Int. 104, 153-163.

Kim, W. Y. (1987). Modelling short-period crustal phases at regional distances for seismic source parameter inversion, Phys. Earth Planet. Interiors 47, 159-178.
Kim, W. Y., D. W. Simpson, and P. G. Richards (1993). Discrimination of regional earthquakes and explosions in eastern United States using high-frequency data, Geophys. Res. Lett. 20, 1507-1510.

Langston, C. A. (1982). Aspects of $P n$ and $P g$ propagation at regional distances, Bull. Seism. Soc. Am. 72, 457-471.

Lilwall, R. C. (1988). Regional mb:Ms, $P g / L g$ amplitude ratios and $L$ spectral ratios as criteria for distinguishing between earthquakes and explosions: a theoretical study, Geophys. J. 93, 137-147.

Murphy, J. R. and T. J. Bennett (1982). A discrimination analysis of shortperiod regional data at Tonto Forest Observatory, Bull. Seism. Soc. Am. 72, 1351-1366.

Pomeroy, P. W., W. J. Best, and T. V. McEvilly (1982). Test Ban Treaty verification with regional data-a review, Bull. Seism. Soc. Am. 72, S89-S129.

Sandvin, O. and D. Tjøstheim (1978). Multivariate autoregressive representation of seismic $P$-wave signals with application to short-period discrimination, Bull. Seism. Soc. Am. 68, 735-756.

Seber, G. A. F. (1984). Multivariate Observations, Wiley, New York, 686 pp.

Shi, Jinghua, W. Y. Kim, and Paul G. Richards (1996). Variability of crustal attenuation in the northeastern United States from $L g$ waves, $J$. Geophys. Res. 101, 25231-25242.

Stump, B. W., F. Riviére-Barbier, I. Chernoby, and K. Koch (1994). Monitoring a Test Ban Treaty presents scientific challenges, EOS 75, 265273.

Taylor, S. R. (1996). Analysis of high-frequency $P g / L g$ ratios from NTS explosions and western U.S. earthquakes, Bull. Seism. Soc. Am. 86, 1042-1053.

Taylor, S. R., M. D. Denny, E. S. Vergino, and R. E. Glaser (1989). Regional discrimination between NTS explosions and western U.S. earthquakes, Bull. Seism. Soc. Am. 79, 1142-1176.

Wang, J. and T.-L. Teng (1995). Artificial neural network-based seismic detector, Bull. Seism. Soc. Am. 85, 308-319.

Lamont-Doherty Earth Observatory

Columbia University

Palisades, New York 10964

(W.Y.K., V.A., A.L.L.-L., P.G.R.)

Department of Earth and Environmental Sciences

Columbia University

Palisades, New York 10964

(A.L.L.-L., P.G.R.)

Manuscript received 13 November 1995 